\title{
Masterplan 2025 der Österreichischen Gesellschaft für Pneumologie (ÖGP) - die erwartete Entwicklung und Versorgung respiratorischer Erkrankungen in Österreich
}

\begin{abstract}
Michael Studnicka · Bernhard Baumgartner · Josef Bolitschek · Daniel Doberer · Ernst Eber · Josef Eckmayr · Sylvia Hartl · Peter Hesse · Peter Jaksch · Eveline Kink · Meinhard Kneussl · Bernd Lamprecht · Horst Olschewski · Andreas Pfleger · Wolfgang Pohl · Christian Prior · Christoph Puelacher · Andreas Renner · Wolfgang Steflitsch • Ingrid Stelzmüller · Helmut Täubl · Karin Vonbank · Marlies Wagner · Felix Wantke · Romana Wass
\end{abstract}

(C) Der/die Autor(en) 2020

Aus Gründen der einfacheren Lesbarkeit wurde im Text das männliche Geschlecht genannt. Wir möchten aber mit gebotenem Respekt darauf hinweisen, dass wir das weibliche Geschlecht und alle Intersexualitäten gleichwertig einbeziehen.

Prim. Univ.-Prof. Dr. med. M. Studnicka ( $\varangle)$ Landeskrankenhaus Salzburg, Universitätsklinik für Pneumologie/Lungenheilkunde, Müllner Hauptstraße 48, 5020 Salzburg, Österreich

Paracelsus Medical University, Salzburg, Österreich m.studnicka@salk.at

Prim. Dr. B. Baumgartner

Abteilung für Pulmologie, Salzkammergut-Klinikum

Vöcklabruck, Vöcklabruck, Österreich

Prim. Dr. med. J. Bolitschek

Abteilung für Pneumologie, Ordensklinikum Linz GmbH

Elisabethinen, Linz, Österreich

DI Dr. D. Doberer · Dr. K. Vonbank

Klin. Abteilung für Pulmologie, Medizinische Universität

Wien, Wien, Österreich

Prim. Univ.-Prof. Dr. E. Eber · PD Dr. A. Pfleger .

M. Wagner, MSc

Univ.-Klinik für Kinder- und Jugendheilkunde, Medizinische

Universität Graz, Graz, Österreich

Prim. Dr. J. Eckmayr

Abteilung für Lungenkrankheiten, Klinikum

Wels-Grieskirchen, Wels, Österreich

Prim. Ass. Prof. Dr. S. Hartl

2. Interne Lungenabteilung, Otto Wagner-Spital, Wien,

Österreich

Dr. P. Hesse

Ordination Dr. Judith \& Dr. Peter Hesse, Schwechat,

Österreich

PD Dr. P. Jaksch

Klin. Abteilung für Thoraxchirurgie, Medizinische

Universität Wien, Wien, Österreich
Prim. Dr. E. Kink, MBA

Lungenabteilung, LKH Graz II - Standort Enzenbach, Gratwein-Straßengel, Österreich

Univ.-Prof. Dr. M. Kneussl

ehem. 2. Medizinische Abteilung mit Pneumologie,

Wilhelminenspital Wien, Wien, Österreich

Prim. PD Dr. B. Lamprecht · Dr. R. Wass

Klinik für Lungenheilkunde, Kepler Universitätsklinikum, Linz, Österreich

Prim. Univ.-Prof. Dr. H. Olschewski

Klinische Abteilung für Pulmonologie, LKH-Univ. Klinikum Graz, Graz, Österreich

Prim. Univ.-Prof. Dr. W. Pohl · Dr. A. Renner Abteilung für Atmungs- und Lungenerkrankungen, Krankenhaus Hietzing, Wien, Österreich

Univ.-Prof. Dr. C. Prior

Ordination Univ.-Prof. Dr. Christian Prior, Innsbruck, Österreich

Dr. C. Puelacher

REHAMED-Tirol GmbH, Innsbruck, Österreich

Dr. med. W. Steflitsch

Wahlarzt-Ordination für Lungenheilkunde, Ollersbach bei Neulengbach, Österreich

PD Dr. I. Stelzmüller

Ordination Doz. Dr. Ingrid Stelzmüller, Salzburg, Österreich

\section{H. Täubl, MScN}

Standort Natters, Pulmologie, LKH Hochzirl-Natters,

Natters, Österreich

Univ.-Doz. Dr.med. F. Wantke

FAZ Floridsdorfer Allergiezentrum GmbH, Wien, Österreich 
Zusammenfassung Mitglieder der Österreichischen Gesellschaft für Pneumologie (ÖGP) beschreiben die erwartete Entwicklung der respiratorischen Gesundheit und zeigen Wege einer patientenorientierten und kosteneffizienten Versorgung für Österreich auf. Methoden: Im November 2017 trifft sich eine Gruppe von respiratorisch tätigen Ärzten, Pflegekräften und Physiotherapeuten, gemeinsam mit Vertretern von Selbsthilfe-Gruppen und Experten für Gesundheitsentwicklung, um die Themen Rahmenbedingungen für Lungengesundheit, Asthma und Allergie, COPD, Mukoviszidose und andere Krankheitsbilder der pädiatrischen Pneumologie, Infektionen in der Pneumologie, Schlafbezogene Atemstörungen, Interventionelle Pneumologie, Thorakale Onkologie, und Orphan Diseases zu bearbeiten.

Ergebnisse: Respiratorische Erkrankungen sind extrem häufig und werden sehr oft durch individuelles Fehlverhalten (Zigarettenrauchen, Überernährung, körperliche Inaktivität) verursacht. Zudem wird für respiratorische Erkrankungen eine Zunahme der Prävalenz, aber eine Abnahme der Hospitalisierungen erwartet. Folgende Maßnahmen sind erforderlich, um den Herausforderungen der Zukunft zu begegnen.

1. Maßnahmen des Screenings und der Fallfindung sollen für Lungenkarzinom und COPD umgesetzt werden.

2. E-Health (Telemedizin, personalisierte Apps) soll vermehrt eingesetzt werden mit dem Ziel das Patientenmanagement $\mathrm{zu}$ erleichtern.

3. Regionale Unterschiede der medizinischen Versorgung können durch E-Health und Harmonisierung der Angebote der Gesundheitskassen reduziert werden.

4. Patientenschulung und Awareness für respiratorische Erkrankungen soll verbessert werden (essentiell für Schlafstörungen, aber auch für andere respiratorische Erkrankungen)

5. Die angebotene Versorgung soll interprofessionell, und auf der Grundlage erkrankungsspezifischer Boards wie bei Tumorboards (z.B. für interstitielle Lungenerkrankungen, Schlaf und Allergie) erfolgen.

6. Die ambulante Rehabilitation im niedergelassenen Bereich kann einen großen Beitrag zur respiratorischen Gesundheit leisten.

7. Das vermehrte Verständnis der Molekularbiologie wird die personalisierte Medizin und zielgerichtete Therapien (z.B. für Asthma, Lungenkarzinom) ermöglichen, aber auch Gesundheitskosten verändern.

Schlüsselwörter Respiratory · Public health • E-health · Prevention

\section{Masterplan 2025 of the Austrian Society of Pneumology (ASP) - the expected burden and management of respiratory diseases in Austria}

Summary Scientific Members of the Austrian Society of Pneumology describe the expected development in respiratory health and provide guidance towards patient-oriented and cost-efficient respiratory care in Austria.

Methods: In November 2017, respiratory care providers (physicians, nurses, physiotherapists) together with patient's advocacy groups and experts in health development, collaborated in workshops on: respiratory health and the environment, bronchial asthma and allergy, COPD, pediatric respiratory disease, respiratory infections, sleep disorders, interventional pneumology, thoracic oncology and orphan diseases.

Results: Respiratory disease is extremely prevalent and driven by ill-health behavior, i.e. cigarette smoking, over-eating and physical inactivity. For the majority of respiratory diseases increased prevalence, but decreased hospitalizations are expected.

The following measures should be implemented to deal with future challenges:

1. Screening and case-finding should be implemented for lung cancer and COPD.

2. E-health solutions (telemedicine, personal apps) should be used to facilitate patient management.

3. Regional differences in respiratory care should be reduced through E-health and harmonization of health insurance benefits across Austria.

4. Patient education and awareness, to reduce respiratory health illiteracy should be increased, which is essential for sleep disorders but relevant also for other respiratory diseases.

5. Respiratory care should be inter-professional, provided via disease-specific boards beyond lung cancer (for ILDs, sleep, allergy)

6. Programs for outpatient's pulmonary rehabilitation can have a major impact on respiratory health.

7. Increased understanding of molecular pathways will drive personalized medicine, targeted therapy (for asthma, lung cancer) and subsequently health care costs.

Keywords Respiratory · Public health · E-health • Prevention

\section{Vorwort}

Im November 2017 traf sich eine Gruppe von Experten verschiedener Professionen (Ärzte, Pflege, Physiotherapeuten) um im Auftrag der ÖGP den Masterplan 2025 für die Entwicklung der pneumologischen Versorgung in Österreich zu erarbeiten.

Im ersten Teil wurde von Vertretern des ÖBIG (Dr. Stefan Mildschuh), der ÖSG (Österreichischer Strukturplan Gesundheit) vorgestellt. Dieser steckt jenes österreich-spezifische Szenario ab, innerhalb dessen der spezifisch pneumologische Masterplan zu entwickeln war.

Der zweite Teil der Veranstaltung bestand darin, dass die Expertengruppen jeweils in interprofessioneller Zusammensetzung, die einzelnen Themengebiete bearbeiteten. Am Ende des Tages erfolgte dann 
die Präsentation und Diskussion dieser Ergebnisse mit Vertretern pneumologischer Selbsthilfegruppen, mit der Möglichkeit die Ergebnisse der Expertenworkshops aus Patientensicht zu kommentieren.

Der nächste Schritt bestand darin, diese Ergebnisse in die Form einer wissenschaftlichen Publikation zu bringen. Ich hoffe Sie haben Freude beim Lesen, und hoffe der Masterplan der ÖGP gibt Orientierung und hilft zu verstehen, wo die Reise der Pneumologie in den nächsten Jahren hingehen soll. Die einzelnen Kapitel folgen dem Duktus: Prävalenz und Krankheitsfolgen, aktuelle Versorgungssituation, erwartete Innovation, Versorgung aus Patientensicht, Szenario 2025 und Patientensicht.

Ich möchte mich bei allen bedanken, die dabei waren, und die in der Nachbereitung dieser Veranstaltung die Geduld nicht verloren haben diesen Masterplan zu verschriftlichen. Nun steht der Bauplan für das „Haus“ der österreichischen Pneumologie 2025. Es liegt an uns, dieses „Haus“ Pneumologie 2025 auch zu bauen.

Univ. Prof. Dr. Michael Studnicka

Präsident der ÖGP 2015-2017

Verantwortlicher für die Umsetzung des Masterplans, im Namen aller Teilnehmer (siehe Autorenliste und Danksagung)

\section{Rahmenbedingungen für Lungengesundheit}

Unsere Gesundheit wird von verschiedenen Faktoren determiniert ([1]; Abb. 1). Diese Faktoren sind einerseits als unveränderbare, individuelle Faktoren wie Genetik, Alter oder Geschlecht bestimmt, andererseits wird unsere Gesundheit im großen Ausmaß von unserem Verhalten bestimmt. Körperliche Inaktivität, Ernährung, Tabak- oder Alkoholkonsum, um nur einige Faktoren aufzulisten, führen im Laufe unseres Lebens zu akuten oder chronischen Erkrankungen. Nicht übertragbare Erkrankungen wie chronische Lungenerkrankungen, Diabetes mellitus, kardiovasku-

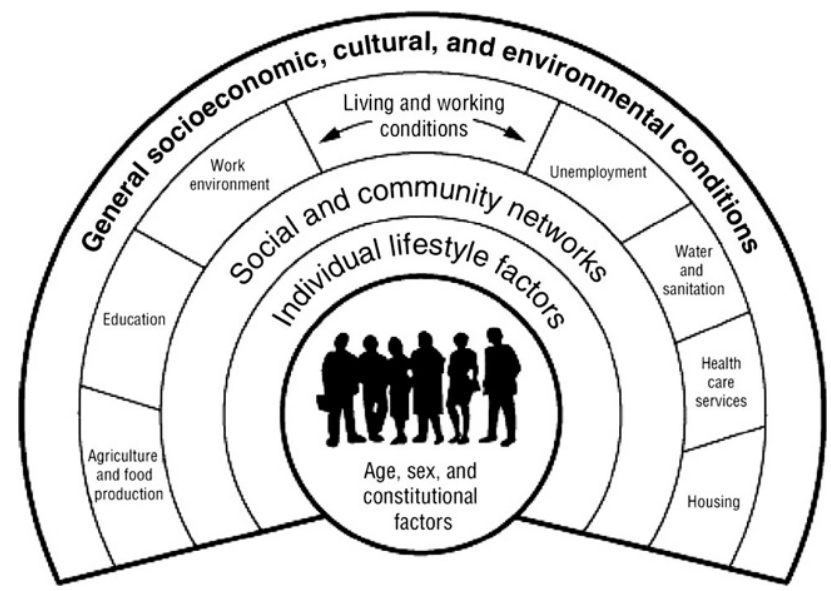

Abb. 1 Modell zur Darstellung der sozialen Determinanten von Gesundheit nach Dahlgren und Whitehead [1] läre Erkrankungen und maligne Erkrankungen werden von der WHO aktuell als größte „Killer“ eingestuft [2].

Die Lunge ist jenes Organ, das am stärksten gegenüber Umweltnoxen, Chemikalien und infektiösen Organismen exponiert ist. Weltweit sind zumindest zwei Milliarden Menschen schädlichen Abgasen und Rauchbelastung, verursacht $u$. a. durch die inkomplette Verbrennung von Biomaterialen, ausgesetzt. Eine Milliarde Menschen inhalieren regelmäßig Zigarettenrauch, und eine weitere Milliarde stark verschmutzte Luft. Armut, Überbevölkerung und schlechter Bildungsstatus sind weitere, zusätzliche Risikofaktoren für Lungenkrankheiten.

\section{Prävalenz und Krankheitsfolgen}

Erkrankungen der Atemwege und der Lunge stellen weltweit ein immenses Gesundheitsproblem dar: fünf Lungenerkrankungen (COPD, Asthma bronchiale, Bronchialkarzinom, Infektionen der unteren Atemwege und Tuberkulose) zählen zu den häufigsten Ursachen für schwere gesundheitliche Beeinträchtigung und Verlust an Lebenszeit.

Allein an COPD leiden weltweit zumindest 65Mio. Menschen, von denen jährlich 3 Mio. versterben. Somit ist COPD die dritthäufigste Todesursache. An Asthma bronchiale leiden geschätzte 334 Mio. Menschen weltweit. Asthma ist die häufigste chronische Erkrankung im Kindesalter mit einer Prävalenz von $10-15 \%$, Tendenz steigend [2].

In Ländern wie Österreich, mit einem hohen Sozio-Demographischen Index (SDI) werden die disability-adjusted life years (DALYs = jene Lebensjahre, die entweder durch „Disability“ oder vorzeitigen Tod im Gefolge einer Erkrankung verlorengehen.) $\mathrm{zu}$ einem Großteil durch Verhaltensweisen bestimmt [3], wie eine Publikation der „Global Burden of Disease Study“ am Beispiel der COPD zeigt (Abb. 2).

Rauchen ist einer der wesentlichen Risikofaktoren für respiratorische Gesundheit. Das Rauchverhalten in

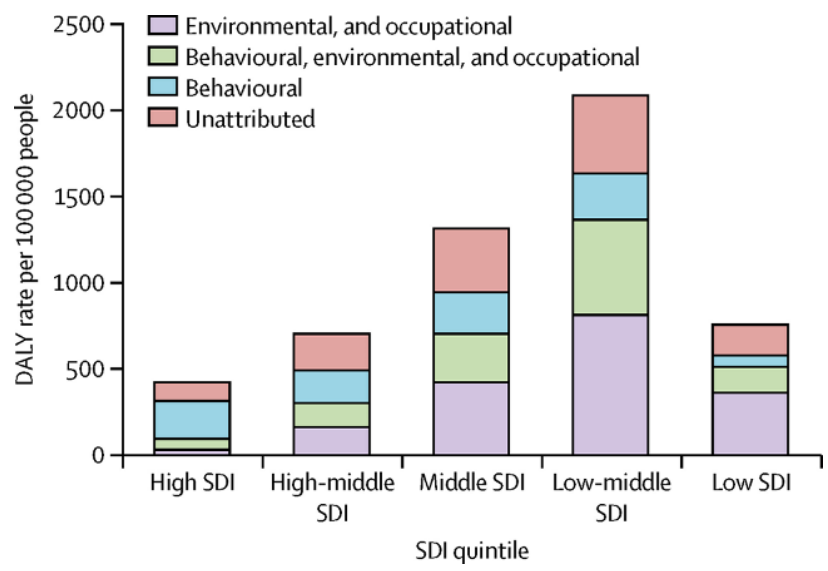

Abb. 2 Beitrag von Verhaltens-, Umwelt- und ArbeitsRisikofaktoren zur DALY am Beispiel der COPD (SDI SocioDemographic-Index) [3] 
Österreich wurde 2008 vom Ludwig-Boltzmann-Institut für Suchtforschung im Auftrag des Bundesministeriums für Gesundheit untersucht: $38 \%$ der österreichischen Bevölkerung rauchen, $43 \%$ der Männer und $34 \%$ der Frauen; wobei $28 \%$ der Österreicher angeben täglich (31\% der Männer und $26 \%$ der Frauen) zu rauchen [4].

Laut WHO sterben in Europa verglichen mit anderen Regionen die meisten Menschen an den Folgen des Tabakrauchens (16\% der Erwachsenentodesfälle). Global wird die Zahl der durch Zigarettenrauch verursachten Todesfälle auf $12 \%$ geschätzt [5]. Zigarettenrauchen ist ein erklärender Risikofaktor für $90 \%$ aller Lungenkarzinomfälle; aktuell sterben weltweit bereits mehr Frauen an einem Lungenkarzinom als an einem Mammakarzinom. Zigarettenrauch ist für geschätzte $80 \%$ der COPD-Todesfälle verantwortlich. Zigarettenrauchen ist auch ein wesentlicher Risikofaktor für die Entstehung und den klinischen Verlauf (vermehrte Exazerbationen) von Asthma bronchiale. Weiters besteht ausreichende Evidenz für einen Zusammenhang zwischen Zigarettenrauchen und dem Auftreten von Tuberkulose [6]. Zunehmend werden auch schädigende Auswirkungen des mütterlichen Zigarettenrauchens auf die intrauterine, embryonale Entwicklung mit negativen Folgen für die Lungenentwicklung berichtet [7].

Neben dem aktiven Rauchen stellt Passivrauchen, im Englischen besser als „second hand smoke“ („Rauch aus zweiter Hand“) bezeichnet, für eine Vielzahl von Lungenkrankheiten einen wesentlichen Risikofaktor dar. Für nahezu alle Erkrankungen, wo aktives Rauchen ursächlich ist, wie bei Lungenkarzinom, COPD und Asthma, um die häufigsten zu nennen, besteht auch ein Zusammenhang mit Passivrauchen [8]. Passivrauchen ist als Luftschadstoff zu beurteilen, und die nicht-rauchende Bevölkerung davor zu schützen. Maßnahmen der gesetzlichen Reduktion von Passivrauchen, z.B. im öffentlichen Bereich der Gastronomie, haben jedenfalls zu einem deutlichen Rückgang bei COPD Exazerbationen geführt [9] .

Die zunehmende, von der Wissenschaft bewiesene, von Politik und Industrie jedoch teilweise bagatellisierte globale Umweltproblematik ist von Menschen verursacht. Die Verschmutzung der Luft und der Meere führt zu dramatischen klimatischen Veränderungen, die ihrerseits direkten Einfluss auf die allgemeine Gesundheit sowie im Speziellen auf die Lungengesundheit haben. Als Folge klimatischer Veränderungen treten einerseits langdauernde Hitzewellen und andererseits extreme Regenfälle mit Überflutungen auf [10].

Der Einfluss dieser klimatischen Veränderungen auf Lungenkrankheiten ist vielfältig und im vollen Ausmaß noch nicht erfasst. Zuallererst werden präexistente Lungenerkrankungen wie COPD und Asthma bronchiale verschlechtert. Die erhöhte Belastung der Außenluft durch Feinstaub, bodennahes Ozon, und Allergene (wie z.B. Pollen und Pilzsporen) führt darüber hinaus zum vermehrten Auftreten von chronisch-obstruktiven Erkrankungen (wie Asthma und COPD) und Infektionskrankheiten der Lunge [11]. Die European Environment Agency (EEA) berichtet in ihrem Report von 2018, dass in der gesamten EU jährlich allein durch Feinstaubbelastung 4.466.000 Lebensjahre verlorengehen; in Österreich sind dies 60.200 Lebensjahre/Jahr [12].

\section{Handlungsplan der ÖGP}

Die Österreichische Gesellschaft für Pneumologie (ÖGP) als wissenschaftliche Fachgesellschaft kann im Zusammenhang mit den genannten Risikofaktoren für eingeschränkte Lungengesundheit in verschiedenster Weise aktiv werden. Neben der Förderung der Ausbildung von pneumologisch tätigen Ärzten, die zunehmend auch für die Herausforderungen von Umweltschutz, Klimawandel und Suchtprävention vorbereitet werden, ist es wichtig, dass die ÖGP ihre Expertise für gesundheitspolitische Entscheidungen zur Verfügung stellt und mit wissenschaftlichen Argumenten gegen mögliche Fehlentwicklungen der Umwelt- und Gesundheitspolitik auftritt.

Von außerordentlicher Bedeutung für die ÖGP ist es auch, sich in Versorgungs- und Präventionskonzepte der Zukunft einzubringen, wobei Präventivmaßnahmen vermehrtes Augenmerk geschenkt werden muss. Die Stärkung der Gesundheitskompetenz muss in Österreich weiterentwickelt werden, und ist ein zentraler Eckpunkt der Gesundheitsreform. Die europäische Kommission betrachtet im Zusammenhang mit Patienten-Empowerment und eHealth, die digitale Gesundheitskompetenz als einen zentralen Entwicklungsschwerpunkt der EU-Gesundheitsstrategie. Zuletzt hat die Weltgesundheitsorganisation (WHO) weltweit Gesundheitskompetenz als zentrale Gesundheitsdeterminante definiert, um die Gesundheit nachhaltig zu verbessern. Die Mitarbeit an Disease-Management-Programmen ist und wird in Zukunft mit der zunehmenden Überalterung von größerer Bedeutung sein, da die Bettenkapazität im Krankenhaus bereits heute am Limit ist.

Wichtig für die ÖGP ist es auch, in Zukunft vermehrt in Projekte und Taskforces mit Arbeitsmedizinern, Epidemiologen, Klimaforschern, Sozialmedizinern und anderen Experten $\mathrm{zu}$ investieren. Weiters sollte die ÖGP in politischen und wissenschaftlichen Gremien die Voraussetzungen schaffen, um auf zukünftige Herausforderungen durch Klimawandel und Umweltverschmutzung bestmöglich vorbereitet $\mathrm{zu}$ sein.

\section{Asthma bronchiale und Allergie}

\section{Prävalenz und Krankheitsfolgen}

Nach Einschätzung österreichischer Experten, stellt sich die Epidemiologie von Asthma und Allergie, wie 
folgt, dar: Fünf Prozent der Erwachsenen und 10\% der Kinder leiden unter Asthma bronchiale. 50\% der Kinder zeigen eine allergische Sensibilisierung, $20 \%$ haben eine klinisch relevante Allergie. $25 \%$ der Erwachsenen zeigen eine allergische Sensibilisierung, $10 \%$ haben eine klinisch relevante Allergie.

\section{Aktuelle Versorgungssituation}

Die Diagnostik von Asthma und Allergie ist in Österreich $\mathrm{zu}$ verbessern, da eine große Zahl von Patienten mit Asthma und Allergie nicht diagnostiziert sind; dies betrifft in erster Linie wenig symptomatische Patienten. Der Übergang der Patientenversorgung bei adoleszenten Asthmatikern von der Pädiatrie zur Erwachsenenpneumologie ist ebenfalls verbesserungswürdig. Die unspezifische Provokationstestung (z. B. Methacholin) erfolgt in der pneumologischen Krankenhausambulanz und im niedergelassenen Bereich mit unterschiedlicher Verfügbarkeit. Zuletzt ist die Diagnostik von schwerem Asthma komplex, und daher sollten Patienten zur exakten Diagnosestellung an ein Asthmazentrum (siehe www.asa-net.at) - zugewiesen werden [13-17].

Nach Expertenmeinung dauert es im Schnitt 7 Jahre, bis ein Allergiker seine Diagnose erhält. Auch die Verfügbarkeit der Allergiediagnostik ist regional unterschiedlich und wird von Fachärzten für Pneumologie, HNO, Dermatologie und Pädiatrie sowie fachspezifischen Spitalsambulanzen wahrgenommen. In Wien, im Burgenland und in Tirol gibt es zusätzlich Allergieambulatorien.

Die hier getroffenen Festlegungen können für Österreich nicht durch Zahlen belegt werden, da diese nicht verfügbar sind [16-20]. Die argumentierten Einschätzungen basieren auf den Erfahrungen der Expertenkommission und einer Abschätzung der Erkrankungshäufigkeiten auf der Grundlage internationaler Daten.

Die Versorgung von Patienten mit Asthma erfolgt im niedergelassenen Bereich einerseits durch Fachärzte für Pneumologie, Pädiatrie [21] oder Innere Medizin als auch durch Allgemeinmediziner. Patienten mit schwerem Asthma sollten soweit verfügbar regelmäßig in Spezialambulanzen gesehen werden. Dennoch gilt die Erkrankung bei über $50 \%$ der Asthmapatienten als „nicht kontrolliert“, wenn man die Definitionen internationaler Empfehlungen zugrunde legt. Diese Patienten sind jedoch klinisch nicht bedroht, wie die stetig fallende Zahl an Asthmatoten belegt [16, 17]. Der hohe Anteil an Patienten mit nicht kontrolliertem Asthma liegt unter anderem daran, dass eine große Anzahl dieser Patienten ihre Situation als „kontrolliert“ interpretiert [22]. Schwere AsthmaExazerbationen sind mittlerweile in Spitalsambulanzen und im stationären Bereich sehr selten und die Mortalität bei Asthma bronchiale geht deutlich zurück.
Im stationären Setting werden Patienten mit Asthma zur Atemphysiotherapie zugewiesen. Es erfolgt eine Schulung, die Inhalationstechniken und Informationen zur Krankheit sowie Notfallstrategien umfasst [23]. Die Zahl der freiberuflich, außerhalb der Kliniken arbeitenden Atemphysiotherapeuten ist gering, daher ist extramural die Versorgung von Asthma Patienten verbesserbar.

Eine Schulung der Asthma Patienten findet im niedergelassenen Bereich nur zum Teil statt. Mit ein Grund dafür ist, dass in Österreich die Finanzierung dieser notwendigen, aber zeitaufwändigen Intervention (= Schulung) nicht gewährleistet ist. Diese Schulungen könnten durch Fachärzte, und auch durch Allgemeinmediziner erfolgen. Um hier ausreichende Effektivität zu erzielen, sind Maßnahmen der Qualitätssicherung und Standardisierung für Schulungsprogramme erforderlich.

Die subkutane spezifische Immuntherapie (SCIT) als auch die sublinguale Immuntherapie (SLIT) wird im niedergelassenen Bereich durchgeführt [24-27]. Allerdings scheitert auch hier eine ausreichende Versorgung an der Remuneration dieser sehr zeitaufwändigen Therapie.

\section{Erwartete Innovation}

\section{Allergie}

Der Pricktest hat weiterhin große Bedeutung als Basis der Typ-1 Allergiediagnostik und kann durch die Serologie nicht ersetzt werden [28]. Serologie und Komponentendiagnostik, insbesondere in Form von Allergenchips, bekommen in Zukunft sicherlich zunehmende Bedeutung. Die Verbesserung der Komponentendiagnostik (z. B. Sensitivität), und die Automatisierung der Herstellung wird mittelfristig zu reduzierten Preisen und zu einer breiteren Anwendung führen. Dadurch wird in Zukunft die Diagnostik erweitert und eine genauere Differenzierung allergischer Phänotypen möglich werden. Gleichzeitig werden die Interpretation der Parameter und die daraus resultierenden Therapieentscheidungen bedeutend komplexer werden und daher einer umfassenderen Ausbildung bedürfen.

\section{Asthma bronchiale}

Durch die Verwendung von Telemedizin und Apps können Asthma Schulung und Medikamentenadhärenz zumindest für einen Teil der Patienten deutlich verbessert werden. Beispiele dazu sind Erinnerungsprogramme zur Medikationseinnahme, elektronische Behandlungspläne (,action plan“) oder Mikrochips in Inhalationshilfen zur Kontrolle der Verwendung der Medikation [29].

Patienten mit schwerem Asthma profitieren nachweislich von einer pneumologischen Rehabilitation, und sollten diese auch angeboten bekommen. Patienten mit leichterem Asthma sollten ihre körperliche Aktivität erhöhen und aktiv und selbstverantwortlich 
zu ihrer Therapie beitragen [30]. Rauchentwöhnung ist auch bei Patienten mit Asthma sinnvoll, und Nikotinkarenz verbessert den Wirkungsgrad inhalativer Steroide.

IT-basierte Neuerungen können durchwegs als positive Hilfsmittel der Behandlung eingestuft werden. Dennoch benötigen Patienten stets den persönlichen Kontakt zu Arzt, Pflege und Atemphysiotherapie. Allerdings gibt es rund um die Telemedizin aus unserer Sicht noch zahlreiche Fragen, etwa zur Machbarkeit, zum Zeitaufwand für den Case Manager und zu den juristischen Konsequenzen der Therapieverantwortung, die noch zu klären sind.

\section{Versorgung aus Patientensicht}

Generelle Forderungen von Patienten, dass Ärzte mehr Zeit haben sollten, mehr zuhören und mehr Empathie zeigen sollten, sind bekannt. Der stete Wunsch nach rascheren Terminen und kürzeren Wartezeiten ist ebenso bekannt und nachvollziehbar, allerdings aufgrund des pauschalierten Honorarsystems in Österreich von ärztlicher Seite derzeit nicht erfüllbar.

In Österreich sind alle Medikamente, die für die Behandlung von Asthma und Allergien notwendig sind, auch für jedermann verfügbar vor allem auch neuere, innovative Medikamente (wie z. B. monoklonale Antikörper).

Die Zukunft der ärztlichen Versorgung wird durch einen Mangel an Fachärzten als auch durch verstärkte regionale Defizite gekennzeichnet sein. Die Zuweisung zu ambulanter Rehabilitation, Atemphysiotherapie oder zu Schulungsprogrammen ist derzeit regional unterschiedlich verfügbar.

\section{Szenario 2025 und Maßnahmen}

Die Prävalenz von Asthma und Allergien wird tendenziell weiter steigen. Eine „One fits all“ Behandlung gilt für Asthma schon lange nicht mehr [31]. Die Therapie wird „personalisierter“ werden und die Therapieoptionen werden zunehmen. Eine verbesserte Phänotypisierung (z.B. durch Komponentendiagnostik) und zusätzliche Biomarker [32, 33] werden die „personalisierte Medizin“ verbessern. Trotz technischer Innovation im Bereich der Telemedizin wird die Behandlung von Patienten weiterhin ein höchst persönlicher und personalintensiver Arbeitsbereich bleiben.

Die primäre Versorgung von Patienten mit Asthma soll weiterhin beim Arzt für Allgemeinmedizin durchgeführt werden. Eine lungenfachärztliche Vorstellung zur Diagnosesicherung soll weiterhin erfolgen. Spezialfälle, wie z. B. für schweres Asthma, sollen weiterhin über Spezial-Ambulanzen in Krankenhäusern geführt werden (Vorstellung z. B. einmal pro Jahr). Der Ausbau einer flächendeckenden ambulanten Rehabilitation für Asthmatiker ist zu forcieren.
Aufgrund der steigenden Prävalenz von Asthma bronchiale und Allergien und der zunehmenden Komplexität von Diagnostik und Therapie ist mit höheren Patientenzahlen zu rechnen. Somit ist entweder eine höhere Anzahl an Fachärzten oder eine höhere Anzahl an ambulanten multiprofessionellen Zentren zu planen. Da oftmals Fachärzte unterschiedlicher Expertise zur Diagnostik und Therapie allergischer Erkrankungen wie Asthma bronchiale erforderlich sind, sind Modelle mit Gruppenpraxen, wo Pneumologen, HNOÄrzte, Dermatologen, Pädiater, Allgemeinmediziner, Physiotherapeuten und Pflegepersonen zusammenarbeiten, anzudenken.

Die Zahl der spitalspflichtigen Asthmapatienten sinkt stetig, da die Diagnostik zunehmend im niedergelassenen Bereich erfolgt. Da auch die Allergiediagnostik weniger häufig im Krankenhaus durchgeführt wird, wird die Ausbildung pneumologischer Fachärzte in Zukunft eine gewaltige Herausforderung darstellen. Auch Allgemeinmediziner, von denen eine Basiskompetenz in der Führung von Patienten mit Asthma und Allergie erwartet werden kann, werden in $\mathrm{Zu}$ kunft weniger Fälle während ihrer Ausbildungszeit im Krankenhaus sehen - vor allem, da in der derzeit gültigen Ausbildungsordnung die Pneumologie kein fixer Bestandteil ist.

Patientenschulung und Patienten-Compliance sind vordringlich, da die Kosten der neuen Medikamente (biologicals) hoch sind und wohl weiter steigen werden. Eine bessere Inhalationstechnik wird auch $\mathrm{zu}$ besserer Asthmakontrolle führen. Strukturierte Patientenschulung kann signifikante Mehrkosten als Folge von Asthma-Exazerbation, Spitalsaufenthalt und Krankenstand minimieren.

Auch niedergelassene Allgemeinmediziner sollten eine Asthmaschulung in guter Qualität durchführen können, müssen aber vorher selbst entsprechend ausgebildet werden. Bei Allgemeinmedizinern liegt ein großer Versorgungsauftrag vor allem außerhalb der Ballungszentren. Eine Asthmaschulung sollte die Themen Medikamente, Inhalationshilfen, Information über die Erkrankung und einen Behandlungsplan (,action plan“) für Patienten umfassen.

\section{COPD}

\section{Prävalenz und Krankheitsfolgen}

COPD ist eine häufige, vermeidbare und behandelbare Erkrankung, die neben Tabakrauch und anderen Luftschadstoffen von sozioökonomischen Faktoren, Ernährungsstatus, körperlicher Aktivität sowie genetischen Faktoren beeinflusst wird.

Weltweit wird die COPD Prävalenz für die Gesamtbevölkerung auf 4-21\% geschätzt [34]. Für Österreich wird die Prävalenz der klinisch relevanten COPD bei den über 40-Jährigen laut BOLD Studie [35] auf 10\% geschätzt, wobei die Prävalenz mit dem Alter zunimmt (Lead-Studie: [36]). Die schweren Formen der COPD 
(GOLD Stadien III und IV) wurden in der BOLD Studie bei $1,4 \%$ der Studienpopulation diagnostiziert. Das Lebenszeitrisiko (life-time risk) an COPD zu erkranken, ist im Vergleich zu anderen Volkskrankheiten sehr hoch.

COPD zählt in den westlichen Ländern zu den häufigen Todesursachen. Die Global Burden of Disease Study prognostizierte, dass COPD im Jahr 2030 weltweit die dritthäufigste Todesursache sein werde. Diese Prognose ist allerdings bereits in den 2010er Jahren eingetreten. Die österreichischen Gesundheitsdaten weisen für COPD für das Jahr 2010 12,6 Todesfälle pro 100.000 aus, mit erwarteter steigender Tendenz in den nächsten Jahren [37].

Der wichtigste Risikofaktor in Ländern der westlichen Welt ist unbestritten das Zigarettenrauchen (siehe Kapitel Rahmenbedingungen für Lungengesundheit). Allerdings weist Österreich im Vergleich, mit europäischen Ländern den höchsten Anteil an Jugendlichen (35,4\% der 17-jährigen Burschen, 30,4\% der 17-jährigen Mädchen) Rauchern aus. Auch zeigt sich im europäischen Vergleich eine nur geringe Reduktion des Raucheranteils in den letzten Jahren. Wie in anderen Ländern bestätigte sich mit $80 \%$ eine hohe Rate an nicht diagnostizierten COPD-Patienten.

Die Gesamtbehandlungskosten von COPD in Österreich wurden für das Jahr 2014 mit 505 Mio. € angegeben, entsprechend $0,15 \%$ des BIP, wobei sich die direkten medizinischen Kosten auf 267 Mio. $€$ belaufen und die indirekten Kosten, das heißt die mit COPD verbundenen Krankenstände, Invalidität und vorzeitige Sterblichkeit mit 238 Mio. € angegeben werden [38].

\section{Aktuelle Versorgungssituation}

\section{Präventionsangebote}

Angebote der Verhaltensprävention haben eine zentrale Bedeutung für die Gesundheit, wobei hierzu vor allem die Tabakprävention zählt. Einen wesentlichen Punkt stellen hierbei gesetzliche Regelungen dar, die den Zugang zu Tabakwaren und den Nichtraucherschutz regeln. Die Gesetze, die in Österreich Nichtraucher schützen sollten, sind im internationalen Vergleich extrem lax. So ist der Schutz der Nichtraucher in der Gastronomie erst seit kurzem umgesetzt. In Österreich gibt es zahlreiche Initiativen zu Tabakprävention und Rauchstopp mit besonderem Schwerpunkt auf Jugendliche. Im Rahmen von Vorsorgeuntersuchungen werden Kurzberatungen zum Nikotinstopp, sowie von Krankenkassen und Pensionsversicherungsanstalten Tabakentwöhnungsprogramme angeboten. Insgesamt ist die Tabakprävention in Österreich sowie das Informationsangebot für Patienten mit COPD deutlich verbesserungsfähig.

\section{Versorgungsangebote}

Über das Versorgungsangebot für COPD Patienten liegen keine gesicherten Daten vor. Im internationalen Vergleich fehlen insbesondere Disease-Management-
Programme für Patienten mit COPD sowie der Aufbau von spezialisierten, nichtärztlichen Gesundheitsberufen, insbesondere spezialisierten Pflegekräften und Atemphysiotherapeuten. In der Versorgung durch niedergelassene Fachärzte für Lungenkrankheiten zeigen sich bundesländerspezifische Unterschiede. Auch gibt es Bezirke, die bislang ohne fachärztliche pneumologische Versorgung auskommen müssen. Akutstationär werden COPD-Patienten $\mathrm{zu} 58 \%$ in Abteilungen für Innere Medizin und $\mathrm{zu} \mathrm{32 \%}$ in pneumologischen Abteilungen behandelt. Mit 310 Krankenhausaufenthalten/100.000 Einwohner/Jahr liegt Österreich im Spitzenfeld der OECD-Länder.

Die Wiederaufnahmerate nach einem Krankenhausaufenthalt wegen COPD liegt bei $40 \%$ innerhalb von 90 Tagen und ist ebenfalls höher als in vergleichbaren Ländern. Die Mortalität in Folge einer stationären Aufnahme wegen COPD liegt mit 6,1\% im vergleichbaren, europäischen Durchschnitt [39].

$\mathrm{Zu}$ den wichtigsten Aufgaben der Primärversorgung zählt die Früherkennung der COPD und damit die Durchführung der Spirometrie bei Patienten mit Risikofaktoren bzw. Symptomen (Husten, Sputum, Atemnot) Derzeit wird die Spirometrie beim Allgemeinmediziner in den Bundesländern Wien, Kärnten und Tirol von den Sozialversicherungsträgern nach wie vor nicht erstattet.

Die pneumologische Rehabilitation ist ein weiterer wichtiger Bestandteil einer umfassenden COPD-Versorgung, und wird für alle Patienten, die mit einer täglichen Inhalationstherapie versorgt werden müssen, empfohlen. In Österreich wird eine Rehabilitationsrate von $15 \%$ erreicht, die weit unter dem europäischen Durchschnitt mit 30 \% liegt. Im Jahr 2010 wurden in Österreich 5126 COPD Patienten stationär und 184 Patienten ambulant rehabilitiert. Somit ergab sich ein ambulanter Rehabilitationsanteil in Österreich von $6 \%$ im Vergleich zur Schweiz mit $20 \%$. Entlassungsprogramme für COPD-Patienten, die eine standardisierte Nachsorge definieren („bundle of care") sind laut den Daten des COPD Audit europaweit in $32 \%$ der Krankenhäuser verfügbar, allerdings in nur $8 \%$ der Krankenhäuser in Österreich.

\section{Erwartete Innovation}

COPD wird heute als chronisch-obstruktive Erkrankung der Atemwege verstanden, die im Erwachsenenalter auftritt. Die Ursache wird in einer chronischen Entzündung gesehen, die meist dem Zigarettenrauchen zugeordnet wird.

Andere Ätiologien der COPD bei Nierauchern werden genauer untersucht werden, und insbesondere die Ursachen der COPD im Kindes- und Jugendalter beschrieben werden [40]. Die technische Innovation wird auch in der Funktionsdiagnostik zu einfacheren Lösungen führen (z.B. Spirometrie App) und damit die Vorhersagbarkeit von akuten Verschlechterungen („Exazerbationen“) verbessern [41]. 
Neue molekularbiologische Konzepte werden bei der COPD zum Einsatz von Biologicals führen und COPD damit möglicherweise zu einer heilbaren Erkrankung machen. Der Einsatz der nicht-invasiven Akut- und Langzeitbeatmung wird durch technische Innovation einfacher, und damit billiger werden, und eine breitere Anwendung erfahren.

\section{Versorgung aus Patientensicht}

COPD-Patienten zeigen ein hohes Interesse an Information über ihre Erkrankung. Diesem Informationsbedarf steht allerdings kein zufriedenstellendes Angebot gegenüber. Ein vermehrtes Schulungs- und Rehabilitationsangebot sowie eine bessere Begleitung nach Entlassung werden als wichtige Maßnahmen angesehen, was durch Patientenbefragungen in Österreich erhoben werden konnte.

\section{Szenario 2025 und Maßnahmen}

- Breit angelegte Tabakprävention mit möglicher Einbeziehung von sozialen Netzwerken, um insbesondere Jugendliche anzusprechen (z. B. YouTube)

- Vermehrte Aufklärungsprojekte in Schulen mit Unterrichtsmaterial für Lehrpersonal

- Ausbau des gesetzlichen Nichtraucherschutzes

- Einführung eines integrierten, patientenorientierten Versorgungsprozesses (Disease-ManagementProgramme) für COPD-Patienten mit Betreuungsalgorithmen für Allgemeinmediziner, Aufbau von Angeboten extramuraler Versorgung sowie Ausbau der nicht-ärztlichen Gesundheitsberufe

- Entlassungsmanagement mit Möglichkeit der zeitnahen Zuweisung nach stationärem Aufenthalt an eine pneumologische Rehabilitationsklinik

- Ausbau der wohnortnahen ambulanten Rehabilitation

- Remuneration der Spirometrie im Rahmen der Vorsorgeuntersuchung zur Früherkennung der COPD bei positivem Raucherstatus (kumulative Tabakrauchbelastung $>10$ pack-years)

- Abänderung der gesetzlichen Definition der Rehabilitation auf „nicht medikamentöse Therapie“, um diese auch bei anderen Sozialversicherungsträger außerhalb der PVA einfordern zu können.

- Abänderung des Ansatzes, dass Rehabilitation nur zur Wiedereingliederung in den Beruf vorgesehen ist, nicht jedoch dazu den Verlauf einer chronischen Erkrankung positiv zu verändern.

- Ausweitung der e-Health Medizin in Behandlungsund Betreuungsalgorithmen bei Patienten mit COPD sowie zunehmende Individualisierung und Personalisierung der COPD Therapie

\section{Mukoviszidose (Cystische Fibrose, CF) und andere Krankheitsbilder der pädiatrischen Pneumologie}

\section{Prävalenz und Krankheitsfolgen bei Mukoviszidose}

Mukoviszidose oder zystische Fibrose ist die häufigste vererbte zum Tod führende Erkrankung der kaukasischen Bevölkerung. Hauptursache ist eine reduzierte Aktivität des cystic fibrosis transmembrane conductance regulator (CFTR)-Proteins, eines Chloridkanals, welcher in vielen Organen des Körpers, so auch in exokrinen Drüsenzellen exprimiert wird.

In Österreich werden pro Jahr 80-100 Neugeborene mit CF (Inzidenz etwa 1:3500), die große Mehrzahl davon mit dem in Österreich seit 1997 bundesweit durchgeführten Neugeborenen-Screening, erfasst. Die definitive Diagnose wird mittels SchweißelektrolytBestimmung und Genetik gestellt. Die jährlich an das Europäische CF Register gemeldeten Daten erfassen in Österreich geschätzt $90 \%$ aller betreuten Kranken. Das waren mit Dezember 2016756 Personen, davon waren 377 (49,9\%) unter, und 379 (50,1\%) über 18 Jahre alt [42]. Derzeit werden die österreichischen CF Patienten in neun pädiatrisch pneumologischen und in drei Erwachsenenzentren betreut. Weitere Erwachsenenzentren sind im Aufbau.

Da CF eine Multiorganerkrankung ist, bedingt dies nach heutigen international anerkannten Standards, dass ein Zentrum eine spezielle CF-relevante Expertise in den Teilgebieten Pneumologie, Infektiologie, Bakteriologie, Mykologie und Resistenztestung, Atemphysiotherapie, Diätologie, Gastroenterologie und Hepatologie, Diabetologie und Endokrinologie, Hals-Nasen-Ohrenheilkunde, Kardiologie sowie Psychologie und in spezieller Krankenpflege ausweisen muss. Zentrumsbezogene Zusammenarbeit mit in CF-Schwangerschaft erfahrenen Gynäkologinnen ist heute Standard.

Aus Sicht der Erkrankten sind der hohe tägliche Zeitaufwand für die Therapie sowie die durch die Notwendigkeit von stationären Behandlungen anfallenden Fehltage in Schule oder am Arbeitsplatz eine große Belastung. Die Betreuung in spezialisierten Zentren wird von den Erkrankten sehr gut angenommen und geschätzt. Die neuen Therapieansätze mit Medikamenten, welche als Korrektoren bzw. Potentiatoren des CFTR-Proteins wirken, geben vielen Patienten und Patientinnen Hoffnung für die Zukunft.

\section{Szenario 2025 und Maßnahmen für Mukoviszidose}

Voraussagen in Europa lassen für 2025 einen Anstieg der unter 18-jährigen Kranken von $50 \%$ und der über 18-jährigen von $75 \%$ erwarten. Die mittlere Lebenserwartung ist durch die verbesserte Behandelbarkeit inklusive der Option einer Lungentransplantation heute in westeuropäischen Ländern, so auch in Österreich, 50 Jahre und darüber ansteigend [43]. 
Damit ergibt sich die Notwendigkeit eines vermehrten Angebots von Erwachsenenzentren mit oben beschriebener Expertise. Wünschenswert und sinnvoll ist die Erfassung aller an CF Erkrankten im Europäischen Register, da auf diesem Wege eine ständige Verbesserung von Therapieangeboten erreicht werden kann.

Die zukünftigen Konzepte „personalisierter Medizin" und die damit einhergehende Lebensqualitätsverbesserung und Lebensverlängerung sind eine Realität, die medizinische Mehrkosten bedeuten wird, allerdings auch indirekte Gesundheitskosten bei verbesserter Erwerbsfähigkeit reduzieren wird. Zukünftige Trends wie über eine Smartphone-Applikation anzuwendende Lungenfunktionserfassung und andere Möglichkeiten mit Übertragung durch spezielle elektronische Methoden (Telemedizin, spezielle Apps, wie Therapieadhärenz-Applikationen) werden breitere Anwendung finden. Angebote für Schulungen sowohl im Pflege- als auch im ärztlichen Bereich sind auszubauen und deren Finanzierung zu sichern.

\section{Mukoviszidose und Lungentransplantation - aktuelle Versorgung}

An der Klinischen Abteilung für Thoraxchirurgie der Medizinischen Universität Wien werden jährlich 100-120 Lungentransplantationen, 60-80 davon an Österreichern durchgeführt, eine geringe Zahl von Lungentransplantationen auch am Transplantationszentrum Innsbruck. Die Mehrzahl der transplantierten Patienten mit CF ist zwischen 25 und 35 Jahre alt. Pro Jahr werden auch 1-2 kindliche bzw. jugendliche CF-Erkrankte transplantiert. Die durchschnittliche Wartezeit liegt derzeit bei 3-6 Monaten. Die Nachbetreuung der in Wien transplantierten Österreicher erfolgt $\mathrm{zu} 80 \%$ in Wien und $\mathrm{zu} 20 \%$ in anderen österreichischen pneumologischen Zentren. Die Zuweisung zur Transplantation erfolgt über eine Vorevaluierung in einem pneumologischen Zentrum. Die in Innsbruck transplantierten Erkrankten werden im Sonderkrankenhaus in Natters weiterbetreut. Aus Patientensicht ist die kurze Wartezeit und geringe Mortalität auf der Warteliste erfreulich. International zählen die Langzeitergebnisse zu den besten weltweit $[44,45]$. Neue Immunsuppressions-Schemata und neue Therapiemöglichkeiten verbessern das Langzeitüberleben.

\section{Mukoviszidose und Lungentransplantation - Szenario 2025}

Die Anzahl der nach einer Lungentransplantation weiter zu betreuenden Patienten wird steigen und damit auch die Notwendigkeit des Ausbaus der Expertise im pneumologisch stationären Bereich. Ausbau von Telemedizin, die weitere Schulung in Posttransplantationspflege und -therapie, wissenschaftliche Fortbildung und Forschung zur weiteren Verbesserung und
Entwicklung neuer Therapieregime wie auch weitere Öffentlichkeitsarbeit sind Aufgaben, mit denen sich die wissenschaftliche Fachgesellschaft weiter befassen wird.

\section{Neurodegenerative und neuromuskuläre Erkrankungen mit Indikation zur Langzeitbeatmung}

Die Konzepte für die Betreuung von jugendlichen Patienten mit invasivem und nicht-invasivem Beatmungsbedarf müssen den wachsenden Erfordernissen angepasst werden. Die Fortschritte der Intensivmedizin, der multimodalen Betreuung sowie der medikamentösen Behandlung im Kindes- und Jugendalter führen dazu, dass die Zahl der neuromuskulär kranken beatmungspflichtigen Patienten im Erwachsenenalter zunimmt. Eine rezente Übersicht zur Langzeitbeatmung bei Kindern und Jugendlichen in Österreich zeigt, dass 12 von 41 pädiatrischen Zentren 143 Patienten betreuen. Davon waren 111 (77,6\%) unter 18 Jahre alt und davon wiederum $44 \%$ neuromuskulär krank; $19 \%$ zeigten eine andere neurologische Grunderkrankung, $9 \%$ eine Atemantriebsstörung, 8\% ein obstruktives Schlafapnoesyndrom, 8\% eine Thorax- und Wirbelsäulenerkrankung, und $12 \%$ andere Erkrankungen mit Beatmungspflichtigkeit [46]. Für 2020 ist von 200 bis 250 Langzeitbeatmeten bzw. atemunterstützten Kindern und Jugendlichen in Österreich auszugehen. Viele Aufgaben der Krankenversorgung sind im derzeitigen Behandlungskonzept den Eltern bzw. betreuenden Angehörigen übertragen, denn über $90 \%$ der erkrankten Kinder und Jugendlichen werden $\mathrm{zu}$ Hause beatmet. Hier besteht ein Bedarf, ein Unterstützungs- und Betreuungskonzept für die betroffenen Familien zu entwickeln.

Die verbesserte Behandlung von Morbus Duchenne und von spinaler Muskelatrophie (SMA), z.B. durch krankheitsmodifizierende Medikamente wie „Nusinersen ${ }^{\circledR}$ “ bei SMA, lassen einen weiteren Anstieg von Kranken mit Langzeitatemunterstützung erwarten. Somit ergibt sich für die kommenden Jahrzehnte ein Mehrbedarf an pneumologischen Erwachsenenzentren mit Beatmungsexpertise.

\section{Primäre ciliäre Dyskinesie (PCD), kongenitale Malformationen des Respirationstrakts, andere seltene angeborene Lungenerkrankungen und Langzeitversorgung von ehemals Frühgeborenen mit bronchopulmonaler Dysplasie (BPD)}

\section{Prävalenz und Krankheitsfolgen}

PCD ist eine meist autosomal rezessiv vererbte Erkrankung unterschiedlich geschätzter Prävalenz (1: 2000 bis 1:40.000) mit Dysfunktion der Zilien des respiratorischen Epithels. Etwa 40-50\% der Betroffenen haben auch einen Situs inversus totalis; umgekehrt haben etwa $25 \%$ der mit einem Situs inversus totalis Geborenen diese Erkrankung. Die Morbidität ab dem Säuglingsalter kann manchmal mit einem 
Atemnotsyndrom bei einem reifen Neugeborenen beginnen, aber auch mit wiederholten Infektionen der oberen Luftwege (Otitiden, später Sinusitiden und vor allem auch Bronchitiden). Diese wiederholten Infekte führen zu sekundärer Keimbesiedelung und nachfolgender Entwicklung von Bronchiektasen. Die Therapie besteht $u$.a. in intermittierender Antibiotikagabe und Atemphysiotherapie. Die Betreuung erfolgt im Kindes- und Jugendalter an pädiatrisch pneumologischen Zentren und im Erwachsenenalter an pneumologischen Kliniken [47].

Von den ca. 10.000 Neugeborenen/Jahr allein in der Steiermark sind 900 bis 1000 vor der 37. Schwangerschaftswoche geboren und somit Frühgeborene. Die hochentwickelte Frühgeborenen-Intensivtherapie und -pflege erlauben es, Frühgeborene bereits ab der 23. Schwangerschaftswoche zu betreuen. Die daraus resultierenden komplexen postpartalen Reifungsprobleme verschiedener Organsysteme betreffen ganz besonders die Lunge mit der Folge der Entwicklung einer chronischen Lungenerkrankung der Frühgeborenen (chronic lung disease of prematurity, CLD bzw. BPD).

Diese Kinder leiden an persistierenden Lungenfunktionsdefiziten und bronchialer Hyperreagibilität ähnlich jenen funktionellen Veränderungen, die bei Asthma bzw. COPD vorliegen [48]. Die Gruppe der seltenen angeborenen Fehlbildungen der Atemwege, des Zwerchfells und des Thorax, wie z.B. Zwerchfellhernien, kongenitale pulmonale Malformationen, und Lungensequester, werden heute meist präpartal erkannt und innerhalb der ersten Lebensjahre einer operativen Versorgung zugeführt, teilweise aber auch weiter bis ins Erwachsenenalter abwartend weiter verfolgt [49].

\section{Szenario 2025 und Maßnahmen}

PCD-Kranke werden in spezialisierten pädiatrisch pneumologischen Zentren betreut und im Erwachsenenalter zunehmend an Erwachsenenpneumologien mit spezieller Expertise weiter zu betreuen sein. Die große Zahl extrem Frühgeborener mit Lungenfunktionsdefiziten, die ins Erwachsenenalter reichen, ist für den niedergelassenen Pneumologen relevant. Nachsorgekonzepte vom Kindes- bis ins Erwachsenenalter sollten in Österreich über die Fachgesellschaften erarbeitet werden. Die bis ins Erwachsenenalter reichende Betreuung von Patienten mit Fehlbildungen der Atemwege und der Lunge sollte ebenfalls durch Konzepte der Fachgesellschaften (ÖGP und Österreichische Gesellschaft für Thoraxchirurgie) abgesichert werden.

\section{Infektionen in der Pneumologie}

Infektiologische Problemstellungen werden auch in den nächsten Jahren und Jahrzehnten einen wesentlichen Teil der Pneumologie ausmachen. Aufgrund der hohen Relevanz dieses Themengebietes erfolgt eine detaillierte Beurteilung für drei Krankheitsbilder: ambulant erworbene Pneumonie (AEP), Bronchiektasen und Tuberkulose.

\section{Ambulant erworbene Pneumonie}

\section{Prävalenz und Krankheitsfolgen}

Die ambulant erworbene Pneumonie (AEP) ist weltweit eine der häufigsten Infektionskrankheiten [50]. Der wesentlichste Risikofaktor „Alter“ ist neben der zunehmenden Erkrankungshäufigkeit auch mit einer hohen Morbidität bzw. Mortalität assoziiert. Nach Peters et al. [51] ist die AEP jene Lungenerkrankung - im Vergleich zu Asthma, COPD, Lungenkrebs und Tuberkulose - mit dem höchsten Anstieg der Erkrankungshäufigkeit bis 2060. Von den EU-weit rund 3,4 Mio. jährlichen Erkrankungsfällen werden 50-80\% ambulant behandelt [52]. Hospitalisiert werden vor allem ältere Menschen mit Komorbiditäten. Die Letalität im stationären Bereich ist mit rund $10 \%$ weiterhin hoch, und hat sich in den letzten Jahren nicht wesentlich verändert. Die EU-weit verursachten Kosten von rund $10 \mathrm{Mrd}$. $€$ pro Jahr werden durch stationäre Behandlung und verlorene Arbeitstage verursacht. Im Gegensatz zu Deutschland ist für Österreich aufgrund fehlender Registerdaten zur AEP eine Berechnung der finanziellen Folgekosten der AEP nicht möglich.

Im Jahr 2016 wurde ein ausführliches Update der S3-Leitlinie zur Behandlung von Erwachsenen mit AEP durch die Deutsche Gesellschaft für Pneumologie (DGP) in Zusammenarbeit mit der ÖGP, der Österreichischen Gesellschaft für Infektiologie und Tropenmedizin (OEGIT) und der Schweizer Gesellschaft für Pneumologie (SGP) publiziert [53]. In den letzten Jahren konnte durch mehrere randomisierte Studien gezeigt werden, dass strukturierte Behandlungswege $\mathrm{zu}$ einem besseren Outcome führen. Als diagnostischer Mindeststandard gilt neben der klinischen Untersuchung der CRB-65-Score (basierend auf Bewusstseinslage, Atemfrequenz, Blutdruck, und Alter), die pulsoxymetrische Sättigung und die Beurteilung von Komorbiditäten. Jeder PneumonieVerdacht muss durch ein Thorax-Röntgen in zwei Ebenen bestätigt werden.

Die 5- bis 7-tägige empirische Antibiose sollte Pneumokokken als häufigsten Erreger immer berücksichtigen. Amoxicillin ( \pm Betalaktamase-InhibitorKombination) stellt die Therapie der Wahl dar. In den nächsten Jahren ist auch bei Pneumonieerregern mit einer veränderten Resistenzlage $\mathrm{zu}$ rechnen. Es empfiehlt sich deshalb die regelmäßige Reevaluation der lokalen mikrobiologischen Resistenzsituation und gegebenenfalls abgeänderte Therapieschemata $\mathrm{zu}$ verwenden. Für die Erregerdiagnostik im stationären Bereich stehen der Legionellen- und PneumokokkenAntigen-Schnelltest im Harn zur Verfügung. Blutkulturen sollten immer vor der antibiotischen Erstgabe erfolgen, diese jedoch nicht verzögern. Die Vitalparameter sollten ebenfalls täglich erhoben werden. 


\section{Szenario 2025 und Maßnahmenkatalog}

Die zunehmende Überalterung der Bevölkerung ist der wesentliche Faktor für die zunehmende Hospitalisierung sowie steigende Morbidität und Mortalität. Um die zumeist langdauernde und kostenintensive Versorgung älterer Patienten im Spital zu reduzieren, wäre eine Versorgung im häuslichen Umfeld bzw. im Alten-/Pflegeheim anzustreben.

Von Bedeutung ist auch die Prävention mit Aufklärung und Impfkampagnen für Influenza und Pneumokokken unter Einbeziehung anderer medizinischer Fachgesellschaften, z. B. der kardiologischen, rheumatologischen, nephrologischen oder infektiologischen Gesellschaft zur Einarbeitung entsprechender Impfempfehlungen auch in deren Behandlungsleitlinien. Ein erster Schritt dazu könnte die Gründung einer Task Force Influenza durch die ÖGP darstellen. Ein weiterer wesentlicher Punkt ist die jährliche Influenza Impfung für alle im medizinischen Bereich tätigen Personen. Strukturierte Behandlungspfade sollten für alle Hausärzte und Notaufnahmen in kurzgefasster Form zur Verfügung gestellt werden, um ein einheitliches und rasches Vorgehen zu ermöglichen. Um die künftig entstehenden Krankheitskosten für Österreich besser abschätzen zu können, sind Pneumonie-Register vergleichbar dem Deutschen CAP-Netz sinnvoll. Solche Register, wenn diese im Längsschnitt betrieben werden, erlauben es, nachzuweisen ob es gelungen ist Versorgungsdefizite zu reduzieren.

Der österreichische Impfplan empfiehlt neben der jährlichen Grippeimpfung die Pneumokokkenimpfung für Menschen ab dem 51. Lebensjahr und für Risikogruppen. Zunächst sollte mit dem 13 valenten Pneumokokken-Konjugat-Impfstoff (PCV13) und mindestens acht Wochen später mit dem 23 valenten Polysaccharid-Impfstoff (PPV23) geimpft werden.

\section{Bronchiektasen}

\section{Prävalenz und Krankheitsfolgen}

Bronchiektasen bezeichnen eine permanente Erweiterung von Bronchien und Bronchiolen mit begleitenden entzündlichen Veränderungen im Bereich der Bronchialwand und des umgebenden Lungengewebes durch eine rezidivierende Inflammation. Die ständige Entzündung bedingt zunächst die Verengung des Bronchialbaumes mit konsekutiver Schleimretention. In weiterer Folge kommt es zur Zerstörung des Epithels, die gestörte mukoziliäre Clearance mit Sekretretention prädestiniert für weitere Infektionen und Inflammationspersistenz. Neben der häufigsten - idiopathischen Form - können Bronchiektasen erworben (z.B. postinfektiös) oder genetisch bedingt sein, aber auch im Rahmen obstruktiver Atemwegserkrankungen auftreten. Durch die breite Verfügbarkeit der hochauflösenden Computertomographie (HRCT) werden Bronchiektasen heute einfacher und deutlich öfter diagnostiziert. Prävalenzdaten zu Bronchiektasen [54] zeigen eine extreme Schwankungsbreite von
2,7/100.000 Einwohner in Finnland bis 52/100.000 in den USA. Dies spricht dafür, dass die Daten entweder nicht in derselben Art und Weise erhoben wurden oder aber unterschiedliche Falldefinitionen verwendet wurden.

Die 2017 publizierten Leitlinien der European Respiratory Society (ERS) zur standardisierten Abklärung [55] empfehlen neben der Bildgebung mittels HRCT zur Beurteilung von Art und Lokalisation der Bronchiektasen folgende weitere Untersuchungen: mikrobiologische Sputum-Untersuchung, Lungenfunktion, Laboruntersuchungen (Blutbild, Differentialblutbild, Immunelektrophorese) sowie Testung auf allergische bronchopulmonale Aspergillose (ABPA). Die Therapie soll laut ERS neben der Behandlung der Grunderkrankung, einer Verbesserung der mukoziliären Clearance und Sekretmobilisation auch die Therapie der Infektion und Inflammation sowie der chronischen Obstruktion beinhalten. Die Einschränkung der Lebensqualität ist bei Patienten mit Bronchiektasen sehr hoch und vergleichbar jener, die für schwere COPD oder schwere Lungengerüsterkrankungen (z. B. IPF) berichtet wird. Bei akuter Exazerbation wird derzeit eine 14-tägige Antibiose empfohlen. Bei einer Neuisolation von Pseudomonas aeruginosa soll die Eradikation angestrebt werden.

\section{Szenario 2025 und Maßnahmenkatalog}

Aktuell erfolgt die Versorgung von Patienten mit Bronchiektasen österreichweit unterschiedlich. Sinnvoll ist der Aufbau von Spezialambulanzen, um eine entsprechende Expertise und kosteneffiziente Patientenversorgung gewährleisten zu können. Im Rahmen von Spezialambulanzen ist neben der Einbringung von $\mathrm{Pa}-$ tienten in Register, wie z. B. das Europäische Register EMBARC [56] oder das deutsche Register PROGNOSIS [57], auch die Teilnahme an Studien möglich.

Die flächendeckende österreichweite Verfügbarkeit der ambulanten Atemphysiotherapie würde eine weitere wesentliche Maßnahme in der Versorgung von Patienten mit Bronchiektasen darstellen. Daten zur Kosteneffizienz dieser Maßnahmen liegen für Österreich nicht vor, zumal die Abrechnungsmodalitäten für die ambulante Atemphysiotherapie je nach Bundesland und Kostenträger variieren. Die weitere Entwicklung und Verfügbarkeit inhalativer Antibioti$\mathrm{ka}$, die vor allem auch aus dem Bereich der Cystischen Fibrose kommen, lässt hoffen, dass in Zukunft vermehrt kosteneffizientere und anwenderfreundlichere Therapieoptionen zur Verfügung stehen werden.

Durch die flächendeckende Verfügbarkeit von CTUntersuchungen und die gesteigerte Awareness für diese Erkrankung wird die Zahl der BronchiektasieDiagnosen vermutlich weiter ansteigen, da sich der Anteil der bislang nicht-diagnostizierten Erkrankungen reduzieren wird. Um eine effiziente Patientenversorgung im niedergelassenen pneumologischen Bereich $\mathrm{zu}$ gewährleisten, kann ein kurzgefasster Leitfaden (Pocket Card) sinnvoll sein. Zudem ist ein ein- 
facher Zugang zu Spezialambulanzen und die ambulante Atemphysiotherapie notwendig, um eine ambulante Versorgung zu ermöglichen.

\section{Tuberkulose}

\section{Prävalenz und Krankheitsfolgen}

Die Tuberkulose gilt mit rund 10 Mio. Erkrankten und 1,8 Mio. Todesfällen weltweit als eine der wichtigsten, weil tödlichsten Infektionskrankheiten. Erfreulicherweise nimmt die welt- als auch europaweite Erkrankungsinzidenz und Mortalität ab [58].

Österreichweit sank die Zahl der jährlichen Neuerkrankungen von 1007 Fällen im Jahr 2005 auf 583 Fälle im Jahr 2015 [59]. Im Jahr 2016 wurden 634 Neuerkrankungen registriert; dieser leichte Anstieg wird mit der seit 2014 gestiegenen Migration aus TBC-Hochprävalenzländern wie Afghanistan, Pakistan, Somalia und den Staaten der russischen Föderation in Verbindung gebracht.

Neben der Erkrankungshäufigkeit ist der Anteil sogenannter resistenter TBC Erkrankungen ein wesentlicher Parameter der Versorgung. Laut Definition spricht man von einer MDR-TBC, wenn der verursachende Tuberkulose-Erreger gegen Isoniazid (INH) und Rifampicin (RMP), die zwei wichtigsten Standardmedikamente, resistent ist. Eine XDR-TBC liegt vor, wenn der verursachende Tuberkulose-Erreger nicht nur gegen INH und RMP sondern auch gegen alle Fluorochinolon-Antibiotika und eines der Aminoglycosid-Antibiotika resistent ist. Sowohl die MDR- als auch XDR-TBC ist mit einer wesentlich längeren Behandlungsdauer und einem höheren Isolations- und Betreuungsaufwand verbunden.

Im Jahr 2016 wurden österreichweit insgesamt 12 MDR- und 2 XDR-Fälle registriert. 2 MDR-Erkrankte stammten aus der österreichischen Nativbevölkerung.

Im Jahr 2017 wurde die neu adaptierte Leitlinie zur Diagnostik und Therapie der Tuberkulose im Erwachsenenalter von der DGP unter Mitwirkung der ÖGP und der Schweizer Gesellschaft für Pneumologie publiziert [60]. Diese Leitlinie bietet eine fundierte Abhandlung sämtlicher mit der TBC in Zusammenhang stehenden Fragen einschließlich MDR- und XDR-TBC, TBC in der Schwangerschaft sowie TBC und HIV.

Derzeit können MDR- und XDR-TBC Fälle in Österreich an den zwei Spezialabteilungen (Wien - Otto-Wagner-Spital, Steiermark - Enzenbach) behandelt werden. Aufgrund der deutlich verlängerten Therapiedauer bei MDR- und XDR-Tuberkulose sind die derzeit vorhandenen 24 Isolationsbetten für Österreich als Minimalversorgung anzusehen.

Das 1968 erlassene österreichische Tuberkulosegesetz wurde zunächst 1992/93 und neuerlich 2017 modernisiert [61], um neben den geänderten epidemiologischen Voraussetzungen auch mit menschenrechtlichen Standards (Stichwort: Bewegungsfreiheit) im Einklang zu stehen. Die bestehende Meldepflicht für klinische TBC Fälle, wurde um die Meldepflicht für Labore erweitert, die am eingelangten Material (z.B. Sputum, BAL) die Diagnose stellen. Weiters ist nun festgelegt, dass die Meldung der TBC innerhalb von drei Tagen nach erfolgter Diagnose an die zuständige Bezirksverwaltungsbehörde $\mathrm{zu}$ erfolgen hat. Die Bezirksverwaltungsbehörde muss jetzt frühestmöglich Patienten, die an Tuberkulose erkrankt sind in einer für den Patienten verständlichen Sprache über die Rechtsfolgen aufklären, die bei einer Verletzung der gesetzlichen Vorschriften zur Isolation zu erwarten sind.

Problematisch ist nach wie vor die teilweise auch in Österreich eingeschränkte Verfügbarkeit von TBCMedikamenten inklusive auch jener der Standardtherapie, z. B. INH. Erfreulicherweise wurden in den letzten Jahren zwei neue Medikamente, nämlich Bedaquilin und Delamanid, zur Behandlung der MDR-TBC zugelassen.

Ein wichtiger Faktor in der Versorgung der TBC sind die Kosten [62]. Betragen die direkten Folgekosten (= Therapiekosten) einer sensiblen Tuberkulose $10.282 €$ pro Patient, so liegen diese bei der MDR-TBC bei $57.213 €$ pro Patient, und sind bei der XDR-TBC mit $170.744 €$ pro Patient nochmals deutlich höher. Neben den erhöhten Therapiekosten kommt hinzu, dass für die MDR- und XDR-TBC neben einer deutlich längeren Therapiedauer auch Aufenthalte in Spezialzentren notwendig sind.

\section{Szenario 2025 und Maßnahmenkatalog}

Neben der gesicherten weltweiten Verfügbarkeit von TBC-Medikamenten kann die Entwicklung von Medikamentenkombinationen („polypills“) die Therapie vereinfachen, und dadurch die Therapie-Adhärenz erhöhen. Vor Behandlungsbeginn ist der Patient bzw. dessen Angehörige oder Betreuung über die Grundprinzipien der Therapie sowie die wesentlichen Arzneimittelnebenwirkungen umfassend und verständlich aufzuklären. Bei Sprachbarriere soll ein Dolmetscher hinzugezogen werden, auch die Verwendung fremdsprachlichen Informationsmaterials ist empfehlenswert.

Die künftige Epidemiologie der TBC in Österreich wird stark durch die Migration aus TBC-Hochprävalenzländern bestimmt und ist deshalb schwer vorauszusagen. Sinnvoll erscheint ein österreichweites Register mit relevanten Informationen (bisherige Behandlung, Medikamente, Resistenzen) um Probleme der Weiterbehandlung bei Wechsel des Bundeslandes zu vermeiden. Auf eine verbesserte sachliche Öffentlichkeitsarbeit (Awareness, aber keine Panikmache) sollte Wert gelegt werden. 


\section{Schlafbezogene Atmungsstörungen}

\section{Prävalenz und Krankheitsfolgen}

Rund ein Drittel der österreichischen Bevölkerung schläft schlecht. Geschätzte 1,2-1,5 Mio. Menschen leiden an chronischen Schlafstörungen. Bis zum Jahr 2025 wird die Zahl der Betroffenen bzw. der Fallfindungen um ca. $30 \%$ steigen [63, 64]. Betroffene und deren engste familiäre Umgebung sind unzureichend über die gesundheitliche Gefährdung durch Schlafstörungen informiert. Die Selbstdetektion einer schlafbezogenen Erkrankung findet kaum statt. Schlafbezogene Atmungsstörungen treten selten als isolierte Einzelerkrankung auf. Der Zusammenhang zwischen nicht erholsamem Schlaf und Bluthochdruck, koronarer Herzerkrankung, Herzrhythmusstörungen, Herzinsuffizienz, pulmonaler Hypertension, obstruktiven bzw. restriktiven Atemwegserkrankungen, Diabetes mellitus, Metabolischem Syndrom, hämatologischen Erkrankungen, Nierenerkrankungen und Erektionsstörungen ist wissenschaftlich evident. Ein interdisziplinäres, fächerübergreifendes Fallfindungs-, Behandlungs- und Therapiekonzept wird derzeit in Österreich noch nicht umgesetzt $[65,66]$.

Gemäß den Empfehlungen zur ambulanten Polygrafie des Arbeitskreises Somnologie der ÖGP 2007 [67] sind derzeit bei weitem nicht alle Indikationen zur Polygrafie ausgeschöpft, z.B. kardiovaskuläre Erkrankungen, nächtliche rhythmologische Ereignisse, nächtlich (non-dipper) oder morgendlich erhöhter Blutdruck, intellektueller Leistungsabfall, erhöhte Tagesmüdigkeit, nächtliche Hypoventilation, neuromuskuläre Erkrankungen und letztlich auch die wichtige präoperative Risikoabschätzung bei Verdacht auf schlafbezogene Atmungsstörung.

\section{Aktuelle Versorgungssituation}

Die Awareness von Ärzten aller Fachgebiete gegenüber schlafbezogenen Atmungsstörungen wird zunehmend besser. Die Fallfindung erfolgt überwiegend im ambulanten Bereich. Es bestehen derzeit keine Wartezeiten für den niederschwelligen Zugang zur kardio-respiratorischen Polygrafie. Die Vergütung der Polygrafie durch die österreichischen Sozialversicherungen (SV) ist österreichweit je nach SV und Bundesland unterschiedlich geregelt. Dadurch bildet sich die schlafmedizinische Fachversorgung im niedergelassenen Bereich unterschiedlich ab. Eine Filterfunktion durch den niedergelassenen Pneumologen mittels einer niederschwelligen Polygrafie existiert nicht in allen Bundesländern [68].

Daten aus Großbritannien lassen erwarten, dass ca. $80 \%$ der Menschen mit OSA nicht diagnostiziert und damit auch nicht behandelt sind [69]. Eine flächendeckende Polygrafie wird damit zu einem Ansteigen der positiven Fallfindung und damit zum weiteren Anstieg der Zuweisungen zur Polysomnografie führen.
Der Zugang zur Polysomnografie, eine überwiegend in einem stationären Schlaflabor durchgeführte Untersuchung, ist im öffentlichen Gesundheitswesen zum Teil mit bis zu 9-monatiger Wartezeit verbunden. Anders als in Deutschland (S3 Leitlinie - Nicht erholsamer Schlaf/Schlafstörungen) gibt es in Österreich keine Leitlinie zur Diagnose und Therapie von nicht erholsamem Schlaf.

Ein interdisziplinäres, fächerübergreifendes Behandlungskonzept [70] findet bisher kaum, am ehesten in Schwerpunktkrankenhäusern, statt. Eine polygrafische Kontrolle nach therapeutischer Beatmungseinstellung ist nur in Ausnahmefällen im niedergelassenen Bereich vorgesehen. Die nichtärztliche Nachsorge (Sleep-Nurse, Rehabilitation, Psychologie, Diätologie) ist bereits im städtischen Bereich schwierig und sinkt im ländlichen Bereich dramatisch [71].

\section{Erwartete Innovation}

Die technische Verbesserung der Diagnostik und Therapie wird $\mathrm{zu}$ einem raschen Ansteigen des $\mathrm{Pa}-$ tientenaufkommens führen. Der diagnostische und therapeutische Flaschenhals bei der Polysomnografie wird in den nächsten Jahren deutlich enger werden. Es ist mit einer Verschärfung der Wartezeiten zu rechnen. Eine Auslagerung in den ambulanten Bereich und telemedizinische Innovationen können Entlastung schaffen. Wie in anderen Fachrichtungen bereits üblich, wird sich ein Schlafboard etablieren, welches über notwendigen Therapien entscheidet. Eine wichtige Innovation wird die Etablierung von spezialisiertem Personal sein. Eine „Sleep-Nurse“, Case- bzw. Disease-Manager können wohnortnah, patientenbezogen, schnell und effizient Nachsorgeleistungen erbringen [72].

\section{Versorgung aus Patientensicht}

Selbsthilfegruppen und Betroffene klagen in erster Linie über die mangelnde Aufmerksamkeit der Öffentlichkeit gegenüber Schlafstörungen. Fehlende Triage und lange Wartezeiten in Diagnostik und Therapie erschweren den raschen Zugang zu medizinischer Hilfe. Aus Sicht der Patienten ist in Österreich eine Behandlungskette nicht abgebildet. Nachsorge und DiseaseManager sowie schlafkompetente medizinische Mitarbeiter fehlen. Ebenso wird der Zugang zu schlafmedizinischen Rehabilitationsmaßnahmen derzeit restriktiv gehandhabt. Ausbildung und Schulung für Betroffene und deren Angehörige finden derzeit kaum statt.

\section{Szenario 2025 und Maßnahmenkatalog (siehe Tab. 1)}

Durch technische Innovationen z. B. tragbare Datenverarbeitung (Wearable Computing) [73] und besseren Informationsstand der Bevölkerung ist für die nächsten Jahre mit einer dramatischen Zunahme der Fallfindung bei OSA in der Größenordnung von zu- 
Tab. 1 Auflistung der Maßnahmen bei schlafbezogenen Atmungsstörungen mit Zuordnung und Zeitfenster

\begin{tabular}{|l|l|l|}
\hline Maßnahme & Wer & Bis wann \\
\hline Öffentlichkeitsarbeit: Medien/Kongresse/Fortbildung & ÖGP & Sofort \\
\hline $\begin{array}{l}\text { Einführung der flächendeckenden adäquat-bezahlten Polygrafie } \\
\text { Abgeltung der biopsychosozialen Anamnese für den Hausarzt } \\
\text { Finanzierung ambulanter schlafmedizinischer Einheiten, } \\
\text { Schaffung schlafmedizinischer Sentinel Einheiten }\end{array}$ & ÖGP & 2020 \\
\hline $\begin{array}{l}\text { Schaffung des Somnologen } \\
\text { Wissenschaftliche Projekte: }\end{array}$ & ÖGP/ÖÄK & Sofort \\
\hline $\begin{array}{l}\text { Erstellung eines Behandlungspfades/einer Leitlinie } \\
\text { Schaffung eines OSAS Registers } \\
\text { Förderung der berufsgruppenübergreifenden Forschung } \\
\text { Konzepte für spezifische Krankheitsbilder und Randgruppen } \\
\text { Österreichischer Kommentar zur DGSM S3 Leitlinie Schlafbezogene Atmungsstörungen (2025) }\end{array}$ & ÖGP Förderung \\
\hline $\begin{array}{l}\text { Optimierung der Versorgung in den Bereichen } \\
\text { Pulmologe als Basisleister }\end{array}$ & \\
\hline $\begin{array}{l}\text { Fächerübergreifende Zusammenarbeit } \\
\text { Förderung von schlafmedizinischen Primärversorgungszentren/Ambulatorien im niedergelassenen Bereich }\end{array}$ \\
\hline $\begin{array}{l}\text { Schlafmedizinische berufsgruppenübergreifende Nachsorge } \\
\text { (Psychologie, Diätologie ...) interdisziplinäre Schlafmedizinische Zentren, Schlafboard (in Anlehnung an Tumorboard) }\end{array}$ & \\
\hline Schlafmedizinische Nachsorge im niedergelassenen Bereich inklusive adäquater Vergütung & ÖGP/ÖÄK \\
\hline Abstimmung zwischen ÖGP anderen Fachgruppen, Forum Pneumologie, Ärztekammer und Selbsthilfegruppen & 2022 \\
\hline Stärkung der Selbsthilfegruppen & - \\
\hline Abbildung Behandlungskette (patientenzentriert) & ÖGP, Forum \\
\hline & Pneumologie \\
\hline & - \\
\hline
\end{tabular}

mindest $30 \% \mathrm{zu}$ rechnen [64]. Die Kombination von kardio-respiratorischer Polygrafie mit Langzeitblutdruckmessung [74] und Langzeit-EKG ergeben völlig neue Einblicke in die Pathophysiologie von Schlafstörungen. Es ist zu erwarten, dass die Versorgung älterer Menschen einen sehr hohen Stellenwert erlangen wird [75]. Schlafmedizinische Abklärung und Therapie statt vorzeitiger Pflegebedürftigkeit werden eine Prämisse werden [76, 77].

\section{Pneumologische Schafmedizin auf dem Weg zur internistisch-pneumologischen Schlafmedizin: Interdisziplinäres Vorgehen in der Schlafmedizin}

Schlafmedizinisch relevante Beschwerden und Komorbiditäten betreffen alle Altersgruppen und steigen im hohen Alter an [77]. Genetische Ursachen, anatomische Veränderungen, protrahierte Schlaffragmentierung und intermittierende Hypoxie führen u.a. zur inadäquaten nächtlichen Sympathicus-Aktivierung. Damit sind chronifizierte Schlafstörungen ein wesentliches Risiko für die Entstehung und Unterhaltung gesundheitsökonomisch wichtiger Krankheitsbilder, zum Beispiel Herz-Kreislauf-Erkrankungen [78], Schlaganfall [65], Demenz [79], Metabolisches Syndrom [80], Krebserkrankungen [81], Dysbiose [82] und Diabetes mellitus [83]. Es zeigen sich auch Gender-assoziierte Unterschiede bezüglich Prävalenz und Krankheitsfolgen [84].

In enger Zusammenarbeit mit nicht-pulmologischen Fachrichtungen ist auf Menschen mit Behinderung, insbesondere mit Beteiligung der oberen Atemwege (z. B. Makroglossie bei Trisomie 21) [85], Menschen mit atemdepressiver Medikation [86], Be- rufskraftfahrer [87], Schichtarbeiter, Menschen mit sexuellen Störungen (Libidoverlust, Impotenz) [88], Menschen nach transitorischer ischämischer Attacke (TIA) oder bereits stattgehabtem zerebralem Insult, und Menschen mit psychiatrischen Erkrankungen [89] besonderes Augenmerk zu legen. Diese beispielhaft erwähnten, medizinisch-fachübergreifenden Verbindungen zeigen auf, dass in Zukunft der Pulmologe eine zentrale Rolle in der interdisziplinären Abklärung und Behandlung von Schlafstörungen, insbesondere von schlafbezogenen Atmungsstörungen, spielen wird.

\section{Ärzteausbildung: Somnologe (Schlafmediziner)}

Um die Anforderungen und die Qualitätssicherung in der Schlafmedizin zu gewährleisten, ist die Etablierung der Schlafmedizin in Österreich eine dringende, längst überfällige Forderung an die Politik. Einerseits braucht es eine flächendeckende Versorgung mit Ärzten in der Primärdiagnostik sowie die umgehende Schaffung des Somnologen.

\section{Informationsstand der Bevölkerung}

Der Bevölkerung ist die Interaktion zwischen Schlaf und chronischer Erkrankung meist nicht bewusst. Ein langer Leidensweg im derzeitigen Gesundheitssystem mit zahlreichen nicht zielführenden Arztbesuchen und schlechtes gesellschaftliches Image von Müdigkeit und Leistungsknick können bei Schlafgestörten zu zusätzlicher Resignation führen. Aufklärung und Informationskampagnen würden die gesundheitlich riskante Situation der betroffenen Menschen deutlich verbessern. 


\section{Von der Primär- bis zur stationären Versorgung}

Es besteht derzeit für primärversorgende Ärzte keine allgemein verpflichtende Ausbildung in Schlafmedizin. Ein Behandlungspfad existiert nicht. Eine weiterführende Abklärung über die medikamentöse Schiene hinaus ist nicht flächendeckend organisiert. Eine schlafmedizinische Ausbildung über eine medikamentöse Behandlung hinaus und die Abgeltung der biopsychosozialen Anamnese durch die SV wäre wünschenswert.

Ambulante Fachversorgung Ein wesentlicher Grund für eine regional unterschiedliche Versorgung liegt in der österreichweit uneinheitlichen Finanzierung durch die SV. Die ambulante Fachversorgung kann stationäre Einrichtungen von nicht behandlungsbedürftigen Fällen entlasten. Mittelfristig soll ein Teil der mit Beatmungsgeräten versorgten Betroffenen in der ambulanten Fachversorgung betreut werden. Dafür sollte die ambulante Fachversorgung als 2. Stufe großzügig ausgebaut und die Polygrafie in die Regelversorgung der SV aufgenommen werden.

Ambulant-stationäre Versorgung In Österreich existieren private Krankenanstalten mit Schlaflaboren als schlafmedizinische Versorger. Diese rechnen überwiegend im Wahlarztsystem ab. Es wird eine Aufwertung des ambulanten schlafmedizinischen Bereiches und die Gleichstellung mit stationären Schlaflaboren in der Abrechnung (z.B. LKF/PRIKRAF), wie bereits in mehreren europäischen Ländern praktiziert, empfohlen [90].

Stationäre Versorgung Für den Zugang zur stationären schlafmedizinischen Versorgung in Österreich existiert derzeit kein Behandlungspfad. Es ist in Österreich noch immer üblich, eine stationäre Abklärung im Schlaflabor, ohne vorangehende ambulante schlafmedizinische Untersuchung durchzuführen. Teilweise werden ambulante Polygrafien stationär durchgeführt [68]. Unter anderem entstehen dadurch bisweilen monatelange Wartezeiten. Eine Triage erfolgt nach medizinischen Kriterien und nach Maßgabe der Verfügbarkeiten.

\section{Innovative Konzepte}

Interdisziplinäre Zusammenarbeit Erstellung eines Behandlungspfades unter Einbeziehung aller Beteiligten. Ziel: ambulante Voruntersuchung und interdisziplinäre Abklärung aller Komorbiditäten, Ausschöpfung aller medikamentösen und ambulanten nichtmedikamentösen Diagnostik- und Therapieformen, Etablierung eines berufsgruppenübergreifenden (z. B. Arzt, Psychotherapeut, Psychologe, Suchtberater, Ernährungsspezialist, Rehabilitationseinrichtung, Sleep-Nurse ...) schlafmedizinischen Primärversorgungszentrums/Ambulatoriums.
Rehabilitationsplan dringliche Aufnahme als REHA-Indikation und in weiterer Folge Evaluierung der Rehabilitation bei nicht erholsamem Schlaf (insbesondere bei Komorbiditäten), schlafmedizinische Abklärung bei allen Rehabilitationsmaßnahmen.

Konzepte für spezifische Krankheitsbilder Schaffung von berufsübergreifenden Steuerungsgruppen, interdisziplinäre Informations-, Diagnose- und Behandlungskonzepte für schlafmedizinische Randgruppen z. B. Trisomie 21.

Versorgungsaufträge Pulmologe in Einzelpraxis als Basisleistung, schlafmedizinisch-spezialisierte Primärversorgungseinheit, Gruppenpraxen, Ambulatorien für spezielle Leistungen, Nachsorge in allen Einheiten.

Qualitätskontrolle Schaffung eines OSAS Registers, Förderung der berufsgruppenübergreifenden Forschung.

\section{Interventionelle Pneumologie}

\section{Einsatzgebiete}

Der Hauptschwerpunkt der Interventionellen Pneumologie ist die Bronchoskopie, und zwar in flexibler und starrer Technik in Lokalanästhesie oder in Allgemeinnarkose. Weitere Schwerpunkte sind die medizinische Thorakoskopie und die Thorakozentese. Die Bronchoskopie kann entweder als diagnostische oder therapeutische Intervention durchgeführt werden, und kommt in erster Linie beim Bronchuskarzinom zum Einsatz. Da pulmonale Läsionen mittels CT früher und damit häufiger erkannt werden, nimmt auch die Zahl der diagnostischen Bronchoskopien zu [91]. Weitere Einsatzgebiete der diagnostischen Bronchoskopie sind die Abklärung bei Verdacht auf ILD mittels BAL und Kryobiopsie, und die infektiologische Abklärung bei Pneumonieverdacht und die Diagnose endobronchialer Pathologien.

Mit verbesserten apparativen Techniken nehmen auch die interventionellen therapeutischen Eingriffe zu (Fremdkörperentfernung, Implantation von Stents, Ventilen, und Coils usw.). Die Implantation von Stents ist als palliative Maßnahme bei Tumorpatienten zur Schienung des zentralen Bronchialsystems indiziert. Die Platzierung von Ventilen oder Coils dient der endoskopischen Volumsreduktion bei hochgradiger Überblähung der Lunge als Folge einer COPD. Die Entfernung von endobronchialen Fremdkörpern (z. B. Aspiration im Gefolge zahnärztlicher Behandlung) ist dagegen von unveränderter Häufigkeit.

Eine weitere Domäne der endobronchialen Intervention stellt die Blutstillung dar. Diese kann Patienten mit Tumorerkrankungen, Patienten mit Cystischer Fibrose und Patienten mit Bronchiektasen betreffen. Hier erfolgt primär eine Inspektion und falls möglich 
lokale Blutstillung und in weiterer Folge oftmals eine CT-Angiographie mit Clipping der Bronchialarterien zur definitiven Blutstillung. Auch für diese Indikationen ist mit einer Zunahme der Häufigkeit zu rechnen, da z. B. die Lebenserwartung von Patienten mit CF deutlich gestiegen ist.

Die Domäne der Thorakoskopie sind sämtliche benignen und malignen Pleuraerkrankungen.

\section{Aktuelle Versorgungssituation}

Die interventionelle bronchoskopische Versorgung ist in Österreich durch Experten der pneumologischen Abteilungen gewährleistet, wenngleich regionale Unterschiede der Erreichbarkeit dieser Kliniken bestehen. Die interventionelle Bronchoskopie erfolgt, da diese im Regelfall in Allgemeinanästhesie durchgeführt wird, zurzeit stationär.

An den pneumologischen Kliniken in Österreich kommen folgende Eingriffe zur Anwendung:

- Stentapplikation bei benignen und malignen endobronchialen Stenosen

- Ultraschall gezielte transbronchiale Nadelaspiration (EBUS-TBNA) zur Diagnose von Malignomen und zum Lymphknotenstaging

- Argongaskoagulation bei Blutungen und Tumorabtragungen

- Kryotherapie zur Rekanalisierung und Tumorabtragung

- Kryobiopsie zur Diagnostik interstitieller Lungenerkrankungen

- Anlage von PleurX-Kathetern bei malignen Pleuraergüssen

- Thorakozentese/Drainage bei Ergüssen

- Endobronchiale Brachytherapie in Zusammenarbeit mit der Strahlenonkologie

\section{Erwartete Innovation}

Zur Verbesserung der Qualität und der Vergleichbarkeit zwischen Zentren sind standardisierte Prozesse erforderlich. Dies betrifft die Ausbildung des Personals, die Materialverarbeitung und den Ablauf von Untersuchungen. Ein wesentlicher Punkt ist auch die digitale Vernetzung; einerseits zwischen den Krankenhäusern und andererseits zwischen Krankenhäusern und niedergelassenen Ärzten. Diese Vernetzung der Bildgebung ermöglicht es Doppelgleisigkeiten und wiederholte Untersuchungen (z. B. bei CT-Thorax) zu vermeiden.

Die On-Site Zytologie, i.e. die zytologische Untersuchung von bronchoskopischen Material während der Untersuchung, führt zu einer Verbesserung der diagnostischen Ausbeute und damit zu einer Beschleunigung der bronchoskopischen Abklärung [92-94].

In naher Zukunft werden Maßnahmen der Ventilimplantation, die Implantation von Coils und die Verwendung von Wasserdampf zur endoskopischen Lun- genvolumenreduktion (BLVR) an Bedeutung zunehmen [95-98].

Die Navigationsbronchoskopie ermöglicht die Diagnostik kleiner und kleinster Rundherde auch in peripheren Lungenabschnitten, und hat das Potential die CT-gezielte Punktion zu ersetzen.

Die periphere Kryobiopsie wird zukünftig die offene oder VATS-gesteuerte Lungenbiopsie bei Verdacht auf ILD ersetzen. Eine weitere Tendenz geht dahin, die diagnostische bronchoskopische Abklärung direkt mit der Therapie zu verknüpfen, wie dies z. B. bei der bronchoskopischen Elektrofrequenzablation der Fall ist.

\section{Versorgung aus Patientensicht}

Folgende Erwartungen an bronchoskopische Eingriffe wurden von Patientenvertretern zur Sprache gebracht:

- Die Bronchoskopie soll schmerz- und komplikationslos sein.

- Die Abklärung soll zeitnahe erfolgen und nur einen kurzen stationären Aufenthalt bedingen.

- Die Aufklärung soll situationsadäquat sein.

- Die Wartezeiten bis zur Befunderstellung sollen möglichst kurz sein, und die Befunde valide (Zweituntersuchungen sollen möglichst vermieden werden.).

- Wenn möglich soll ein ärztlicher Ansprechpartner für Aufklärung, Durchführung und Befundbesprechung zur Verfügung stehen.

\section{Szenario 2025 und Maßnahmen (siehe Tab. 2)}

Der Bedarf an interventionellen Eingriffen und Untersuchungen ist durch eine Zunahme der Erkrankungen und durch neue, therapeutische Methoden stetig steigend. Insbesondere im Gefolge der Etablierung eines Lung Cancer Screening Programms in Österreich in den nächsten 10 Jahren, ist nochmals mit einer deutlichen Zunahme von bronchoskopischen Abklärungen zu rechnen. Dementsprechend ist auch ein erhöhter Personal- und Kostenbedarf zu erwarten. Das Ziel einer patientenorientierten Medizin muss es sein, in möglichst kurzer Zeit eine Abklärung anzubieten, durchzuführen und mit einer Therapieempfehlung abzuschließen („fast track“). Komplexe Interventionen werden vor allem aufgrund möglicher Komplikationen weiterhin stationär oder tagesklinisch abgewickelt werden. Wünschenswert wäre ein Register aller durchgeführten Interventionen (z.B. Stent- oder Ventilimplantationen). Der Maßnahmenkatalog umfasst Aufnahmen der Leistungen in den LKF-Katalog, Standardisierung der Abläufe, digitale Vernetzung und Fortbildungsangebote. 
Tab. 2 Auflistung der Maßnahmen der interventionellen Pneumologie mit Zuordnung und Priorität

\begin{tabular}{|c|c|c|c|}
\hline MASSNAHME & WER & BIS WANN & PRIOR \\
\hline Aufnahme interventioneller Eingriffe in den LKF Katalog (Ventile, Coils, EBUS TBNA) & ÖGP/AK & Ehest & 1 \\
\hline $\begin{array}{l}\text { Standardisierung von } \\
\text { Ausbildung } \\
\text { Dokumentation } \\
\text { Aufklärung } \\
\text { Materialverarbeitung } \\
\text { Befundung } \\
\text { Patientenbesprechung }\end{array}$ & $\begin{array}{l}\text { ÖGP/AK } \\
\text { Träger }\end{array}$ & Laufend & 2 \\
\hline Digitale Vernetzung zwischen allen Trägern und niedergelassenen Ärzten & Träger, Länder, Bund & Ehestmöglich & 1 \\
\hline Positive PR für alle Berufsgruppen & ÖGP & - & - \\
\hline Fortbildungen, v. a. auch fachübergreifend (Patho, Radio, Anästhesie ...) & ÖGP & Laufend & 2 \\
\hline Aktives Anbieten von Kursen (inkl. Basisskills) & ÖGP & Laufend & - \\
\hline
\end{tabular}

\section{Thorakale Onkologie}

\section{Prävalenz und Krankheitsfolgen}

4000-4500 Menschen erkranken in Österreich jährlich an Lungenkrebs. Den Hauptrisikofaktor stellt dabei das Rauchen dar. Es ist davon auszugehen, dass die Erkrankungszahlen bis zum Jahr 2025 weiter ansteigen werden. Man kann mit einer Steigerung um bis zu $25 \%$ rechnen [99]. Den leicht rückläufigen Erkrankungszahlen bei Männern stehen deutlich steigende Erkrankungszahlen bei Frauen gegenüber. Dies ist dem geänderten Rauchverhalten von Frauen in den vergangenen Jahrzehnten geschuldet. Da Lungenkrebs mit rund 4000 Todesfällen pro Jahr auch die höchste Mortalität aller onkologischen Erkrankungen aufweist, ist die individuelle und sozioökonomische Last dieser Erkrankung enorm.

\section{Aktuelle Versorgungssituation}

Derzeit erfolgt die Versorgung von Lungenkrebspatienten vorwiegend stationär oder tagesklinisch. Es ist jedoch davon auszugehen, dass in Zukunft die Betreuung stärker in den ambulanten Bereich rücken wird. Ein Screening-Programm für Lungenkrebs ist in Österreich noch nicht etabliert, daher werden derzeit viele Patienten im niedergelassenen Bereich, beispielsweise im Rahmen von akuten Exazerbationen aufgrund einer COPD oder einer Pneumonie, erstdiagnostiziert. Telemedizinische Konsile werden bereits vereinzelt durchgeführt. Dies wird in der Zukunft deutlich zunehmen. Konkret könnte dies bedeuten, dass sich Patienten im niedergelassenen Bereich vorstellen und die niedergelassenen Kollegen mit einer Klinik bzw. spezialisierten Organisationseinheit per Videokonferenz Kontakt aufnehmen, um Bilder und Befunde zu diskutieren und eine unmittelbare Rückmeldung bezüglich der weiterführenden Abklärung zu erhalten.

Pneumologen sind die optimalen "Case-Manager“ der Lungenkrebspatienten und wollen bzw. sollen diese Aufgabe auch weiterhin wahrnehmen. Von der Diagnosestellung bis zur Palliation können Pneu- mologen die Betreuung von Lungenkrebspatienten umfassend erfüllen. Neben der ärztlichen Betreuung nehmen auch andere Gesundheitsberufe eine zentrale Rolle in der Versorgung von Lungenkrebspatienten ein. Die mobile Pflege ist immer noch unterrepräsentiert. Gut ausgebildete Pflegekräfte könnten im häuslichen Umfeld die Versorgung optimieren und somit Krankenhausaufenthalte oder Arztkonsultationen vermeiden helfen.

Obwohl onkologische Rehabilitation deutlich zur Resilienz beiträgt, ist das Angebot an ambulanten, wohnortnahen onkologischen Rehabilitationseinrichtungen nicht ausreichend und muss ausgebaut werden. Auch die Physiotherapie spielt in der Betreuung von Lungenkrebspatienten derzeit eine untergeordnete Rolle. Während stationärer Aufenthalte bleibt oft zu wenig Zeit für physiotherapeutische Maßnahmen, weshalb die Patienten diesbezüglich häufig unterversorgt sind.

Die Palliativversorgung von Lungenkrebspatienten ist regional sehr unterschiedlich ausgebaut. So stehen Patienten im städtischen Bereich oft Tageshospize und stationäre Hospizeinrichtungen zur Verfügung. Gerade für Patienten aus ländlichen Regionen sind Angebote wie mobile Palliativteams von zentraler Bedeutung. Auch Angebote wie Telefonsupport könnten zielführend sein und sollte erwogen werden.

\section{Erwartete Innovation}

Gegenwärtig stellt sich die Frage, ob durch ein flächendeckendes Lungenkrebsscreening die individuelle und sozioökonomische Bürde von Lungenkrebs verringert werden könnte. Aktuelle Untersuchungen zeigen, dass die „number needed to screen“, also jene Anzahl an Menschen, die untersucht werden müssen, um tatsächlich eine Lungenkrebserkrankung vorteilhaft früh zu diagnostizieren, bei 300 liegt. Im Vergleich dazu liegt diese bei Brustkrebs bei 1000. Die Evidenz und Effizienz eines Screenings sind entsprechend der publizierten Daten für das Lungenkarzinom deutlich besser einzuschätzen als z. B. für das Mammakarzinom. 
Rund 6,5\% der erwachsenen Bevölkerung würden der klassischen Risikogruppe (55-75 Jahre, >30 packyears, aktiver Raucher oder Rauchstopp $<15$ Jahre), die von einer solchen Screening-Untersuchung laut den Daten des NLST (National Lung cancer Screening Trial) profitiert, entsprechen. Erfahrungen anderer Screening-Untersuchungen zeigen, dass ungefähr die Hälfte der Personen der Risikogruppe das Angebot annehmen würde. Allein durch diese Maßnahme könnte die Lungenkrebsmortalität um $20 \%$ reduziert werden [100]. Das entspricht der Mortalitätsreduktion, die durch 7 Jahre Nichtrauchen erreicht werden kann. Eine Kombination aus 15 Jahre Nichtrauchen und Screening würde die Mortalität sogar um $38 \%$ senken [101, 102].

In Ergänzung zum NLST wurde Anfang 2020 die europäische NELSON Studie publiziert, die sich ebenfalls der Fragestellung des Lungenkarzinomscreenings widmet, und ebenfalls eine Mortalitätsreduktion nachweisen konnte [103]. Auf der Grundlage dieser Daten ist es nun an der Zeit auch in Österreich Screeningprogramme zu implementieren.

Innovative Methoden erwarten wir sowohl in der Diagnostik als auch in der Therapie thorakaler Malignome von molekularpathologischen Untersuchungen wie Next Generation Sequencing aus der Liquid Biopsy über zielgerichtete molekulare Antikörpertherapien und (Kombinations-)Immuntherapien [104]. Durch hoch wirksame Therapie und steigende Inzidenz bei sinkender Mortalität wird die Krankheitslast zunehmen und Lungenkrebs für manche Patienten zu einer chronischen Erkrankung werden.

\section{Versorgung aus Patientensicht}

Aus Patientensicht sind in Österreich die rasche Abklärung und der ebenso rasche Zugang $\mathrm{zu}$ Therapien hervorzuheben. Des Weiteren befinden wir uns in Österreich derzeit (noch) in der Situation, dass jeder Patient unabhängig seines Versicherungsstatus uneingeschränkte Diagnostik und Therapie erhält. Durch interdisziplinäre Tumorboards ist der Patient kompetent beraten, und die Entscheidungsverantwortung wird im Team getragen. Gerade im sehr sensiblen onkologischen Bereich wünscht sich der Patient einen Arzt seines Vertrauens und/oder kontinuierlichen Ansprechpartner - eine Aufgabe, die in Zeiten straffer Klinikorganisation und des Arbeitszeitgesetzes oft schwer zu erfüllen ist.

\section{Szenario 2025 und Maßnahmenkatalog}

Im Jahr 2025 werden uns steigende Erkrankungszahlen und eine höhere Krankheitslast vor Herausforderungen insbesondere in Hinblick auf damit verbundene steigende Kosten und die Frage nach der Finanzierbarkeit stellen. Im Jahr 2017 entfallen in Österreich rund $6,5 \%$ der Gesundheitsausgaben auf die Behand-
Tab. 3 Auflistung der Maßnahmen der thorakalen Onkologie mit Zuordnung und Zeitfenster

\begin{tabular}{|c|c|c|}
\hline Maßnahme & Wer & $\begin{array}{l}\text { Bis } \\
\text { wann }\end{array}$ \\
\hline Öffentlichkeitsarbeit (Rauchverbot) & ÖGP & Sofort \\
\hline Evaluierung eines Screening-Programms & - & 2020 \\
\hline $\begin{array}{l}\text { Sensibilisierung für Berufskrankheiten, frühzeitige } \\
\text { Meldung }\end{array}$ & - & 2020 \\
\hline $\begin{array}{l}\text { Wissenschaftliche Projekte im Rahmen der ALCG } \\
\text { Investigator initiated trials } \\
\text { Publikationen } \\
\text { Register }\end{array}$ & $\begin{array}{l}\text { ÖGP, } \\
\text { ALCG }\end{array}$ & 2020 \\
\hline $\begin{array}{l}\text { Optimierung der Versorgung in den Bereichen } \\
\text { Physiotherapie } \\
\text { Onkologische Rehabilitation } \\
\text { Logopädie } \\
\text { Ergotherapie } \\
\text { und deren adäquate Vergütung im niedergelassenen } \\
\text { Bereich }\end{array}$ & - & 2020 \\
\hline $\begin{array}{l}\text { Professionalität der onkologischen Pflege erhöhen } \\
\text { (fachspezifische Aus-Weiterbildungen mit onkologi- } \\
\text { schem Schwerpunkt) }\end{array}$ & - & 2021 \\
\hline $\begin{array}{l}\text { Onkologische Nachsorge im niedergelassenen Be- } \\
\text { reich inklusive adäquater Vergütung }\end{array}$ & - & 2025 \\
\hline $\begin{array}{l}\text { Gute Abstimmung zwischen ÖGP, Forum Pneumolo- } \\
\text { gie, Ärztekammer und Selbsthilfegruppen }\end{array}$ & $\begin{array}{l}\text { ÖGP, } \\
\text { Forum } \\
\text { Pneumolo- } \\
\text { gie }\end{array}$ & 2019 \\
\hline $\begin{array}{l}\text { Lobbying für Finanzierbarkeit kostenintensiver Thera- } \\
\text { pien in Gegenwart und Zukunft }\end{array}$ & ÖGP & 2020 \\
\hline $\begin{array}{l}\text { Spezialisierungen und zertifizierte Lungenkrebszen- } \\
\text { tren }\end{array}$ & ÖGP & 2020 \\
\hline $\begin{array}{l}\text { Ausbau von mobiler Pflege und mobilen Palliativ- } \\
\text { teams }\end{array}$ & - & 2020 \\
\hline
\end{tabular}

lung von Krebserkrankungen. Die Hälfte der Ausgaben entfällt dabei auf den Spitalsbereich [105].

In Folge der teilweise dramatisch verlängerten Lebenszeit durch neue Therapien, als auch in Folge der Umsetzung eines evidenz-basierten Lungenkarzinomscreenings ist mit einer Zunahme pneumologisch-onkologischer Patientenzahlen $\mathrm{zu}$ rechnen. Diese Situation wird vermehrt ambulante und tagesklinische Versorgungsplätze brauchen.

Die Sicherstellung der Finanzierbarkeit onkologischer Therapien ist eine Herausforderung für die Zukunft. Dieser Herausforderung muss sich das österreichische Gesundheitssystem stellen. Mangelhafte Maßnahmen in der Prävention (z.B. Rauchen in der Gastronomie) werden - mit einer Zeitverzögerung von 20 Jahren - einen höheren Ressourcenbedarf in der Behandlung bedeuten.

Die Zukunft der Versorgung von Lungenkrebspatienten wird durch Zentrumsbildungen, weitere Spezialisierungen und Leistungskonzentration geprägt sein (Tab. 3). Damit verbunden ist auch die Sicherung von Qualitätsmerkmalen in der onkologischen Versorgung durch die Zertifizierung von Lungenkrebszentren und die Etablierung von Tumorregistern. Insbesondere auch im Wettbewerb mit internistischen Onkologen muss die vorhandene Expertise erkennbar nach außen getragen werden. 


\section{Orphan Diseases (seltene Krankheiten) mit Lungenbeteiligung}

\section{Prävalenz und Krankheitsfolgen}

Laut Orphanet [106] gibt es in Österreich 400.000 Patienten mit einer „orphan disease“. Dieser Begriff ist definiert als eine Krankheit mit einer Prävalenz unter 1:2000 bei den in der Europäischen Union lebenden Menschen. Die Entwicklung von spezifischen Medikamenten für „orphan diseases“ ist schwierig und kostenintensiv, weil die Rekrutierung von geeigneten Patienten für klinische Studien viel Zeit erfordert und nach der Zulassung eines Präparates die Zahl der verordneten Packungen stets klein bleibt. Daher haben die Zulassungsbehörden ein erleichtertes Zulassungsverfahren für Medikamente mit „orphan drug“ Status eingerichtet (orphan drug designation).

Viele der seltenen Krankheiten betreffen die Lunge, wobei die über 150 teils extrem seltenen interstitiellen Lungenkrankheiten einen hohen Anteil haben. Im Bereich der häufigen Lungenkrankheiten, wie Asthma und COPD, gibt es aber auch seltene bis sehr seltene Subtypen, die einen eigenständigen Phänotyp darstellen. Genannt sei das Asthma, das gemeinsam mit Polyposis nasi auftritt, oder das late/onset asthma [92] oder der pulmonal vaskuläre Phänotyp der COPD [107]. Diese Subtypen entsprechen, gemessen an der Prävalenz, wieder den Kriterien einer „orphan disease“.

Eine tabellarische Übersicht über diese seltenen Lungenkrankheiten wurde von der Swiss Group for Interstitial and Orphan Lung Diseases rezent publiziert (siehe Tab. 4; [108]).

\section{Aktuelle Versorgungssituation}

Bei den meisten „orphan diseases“ werden derzeit lediglich symptomatische Maßnahmen empfohlen, beispielsweise die Langzeitsauerstofftherapie (LTOT) bei Hypoxämie oder die unspezifische Schmerztherapie, ohne dass die eigentliche Ursache der Beschwerden therapeutisch adressiert werden kann.

Für manche seltene Krankheiten gibt es stark pathophysiologisch oder klinisch motivierte Empfehlungen für Medikamente, die bereits in anderen, meistens häufigeren Indikationen zugelassen sind. Das gilt etwa für die Behandlung der Alveolitis bei Sklerodermie mit Cyclophosphamid [109]. Cyclophosphamid ist als Chemotherapeutikum und Immunsuppressivum seit vielen Jahrzehnten in Verwendung. Ein weiteres Beispiel ist die sehr seltene Lymphangioleiomyomatose (LAM), für welche aufgrund von kleinen Fallserien der mTOR-Inhibitor Sirolimus empfohlen wird [110]. Sirolimus (Afinitor ${ }^{\circledR}$ ) wurde erstmals 1999 für die Prophylaxe der Organabstoßung nach Nierentransplantation zugelassen. Weiters wurde Sirolimus für seltene Formen von Brustkrebs und für Tumoren und Epilepsie im Rahmen einer Neurofibromatose zugelassen.
Tab. 4 List of rare lung diseases

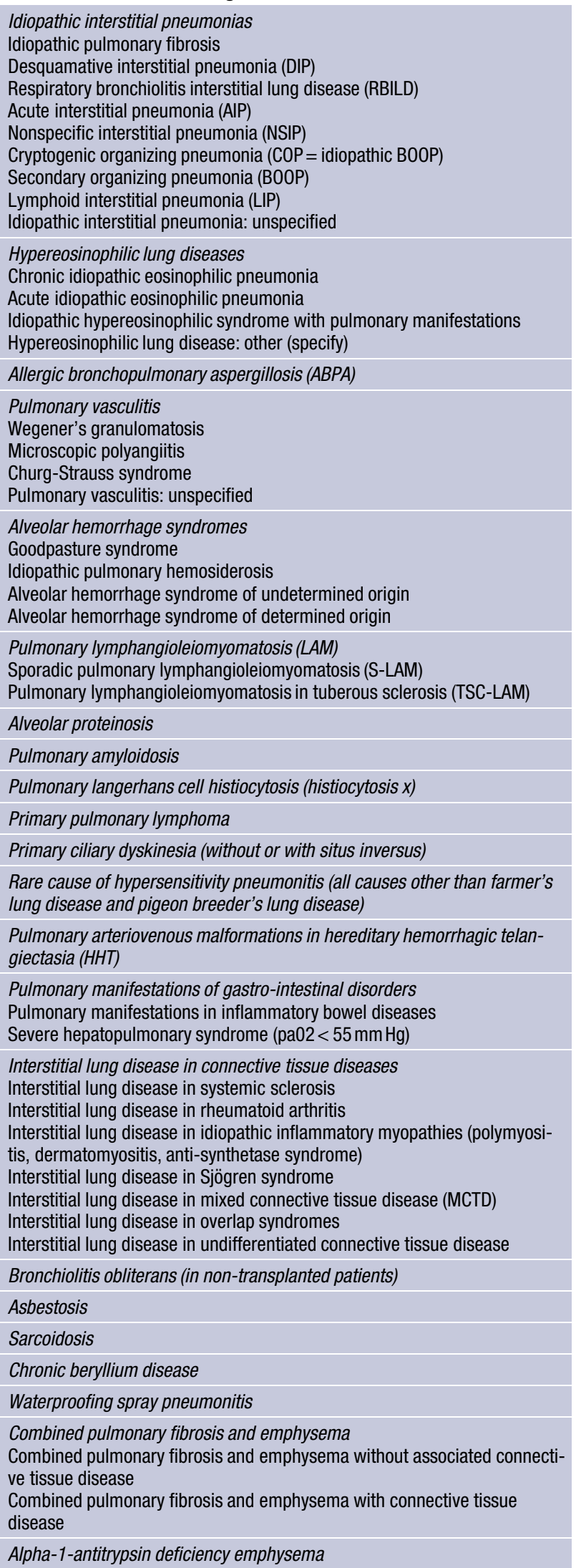


Ein anderes Beispiel ist die Indikationserweiterung von Everolimus (Certican ${ }^{\circledR}$ ). Der mTOR-Inhibitor Everolimus war 2003 ebenfalls für die Prophylaxe der Abstoßungsreaktion nach Nierentransplantation zugelassen worden. Später gab es eine Indikationserweiterung für die Lebertransplantation. 2016 erfolgte darüber hinaus die Zulassung für die sehr seltenen Neuroendokrinen Tumoren (NET) der Lunge und des Gastrointestinaltraktes.

Basierend auf den pathologischen Befunden bei Alveolarproteinose, wo sich die Alveolen der Lunge mit Surfactant Material füllen und so ihre Funktion verlieren, wurde die Behandlung mit wiederholten bronchoalveolären Lavagen etabliert, obwohl dazu nie eine kontrollierte Studie durchgeführt wurde. In den letzten Jahren wurde versucht, gezielt in die pathologischen Mechanismen der Alveolarproteinose einzugreifen, die stets zu einer Inaktivierung von Granulozyten-Kolonie stimulierendem Faktor (G-CSF) und so zur Schwächung der Alveolarmakrophagen führen [111]. G-CSF ist in anderer Indikation zugelassen und damit eine therapeutisch verfügbare Substanz.

Für einen kleinen Teil der vielen „orphan diseases“ wurden neue spezifische Medikamente entwickelt. $\mathrm{Zu}$ den ersten Beispielen zählt die Substitutionstherapie mit Alpha-1-Antitrypsin (AlAT) beim genetisch determinierten A1AT-Mangelsyndrom mit Lungenemphysem. Diese pathophysiologisch begründete Therapie wird bereits über viele Jahre hinweg durchgeführt, auch wenn keine hochwertige Evidenz für Wirksamkeit und Sicherheit vorliegt.

In den letzten Jahren wurden aber auch einige neue, hochwertige Studien mit speziell entwickelten Medikamenten für bestimmte „orphan diseases“ durchgeführt. Alle diese Medikamente machten sich den „orphan drug status“ zu Nutze. Teilweise wurden mit den so zugelassenen Substanzen enorme Gewinne erwirtschaftet. Als Beispiele aus dem pulmonalen Bereich seien die gezielten PAH- bzw. CTEPH-Medikamente genannt, die alle seit 2003 zugelassen wurden. Dazu zählen drei verschiedene Prostanoide, zwei Phosphodiesterase-Inhibitoren (PDE5I), drei Endothelinrezeptorantagonisten (ERA), ein Stimulator der löslichen Guanylatcyclase (sGC), und ein nichtprostanoidischer Stimulator der IP-Rezeptoren (PCA) [112].

Auch für die seltene idiopathische Lungenfibrose (IPF) wurden zwei speziell entwickelte Medikamente zugelassen (Pirfenidon und Nintedanib) [113]. Sie haben zu einer spürbaren Verbesserung der Prognose geführt, obwohl viele Details zum Wirkmechanismus noch unklar sind [114].

In die gleiche Kategorie $\mathrm{zu}$ zählen ist auch eine Subgruppe von bösartigen Lungentumoren. Es handelt sich dabei um Tumoren, die aktivierende Mutationen innerhalb ihrer zellulären Wachstumssignale aufweisen und auf spezifische Inhibitoren (Tyrosinkinaseinhibitoren, TKI) ansprechen [115, 116]. Diese Medikamente führen zu deutlich besseren Therapie- effekten als eine herkömmliche Chemotherapie und haben vergleichsweise geringe Nebenwirkungen. Wie zu erwarten war, sind alle TKIs sehr hochpreisige Medikamente.

Ein Medikament mit aktuell besonders hohem öffentlichem Interesse ist Nusinersen (Spinraza ${ }^{\circledR}$ ) für die spinale Muskelatrophie beim Kind, welches Kosten von ca. $€ 500.000 \mathrm{im}$ ersten Therapiejahr und $€ 250.000$ in jedem weiteren Jahr verursacht [117, 118]. Unter Therapie entwickeln sich die Kinder nach gegenwärtigem Kenntnisstand normal und sollten theoretisch auch eine normale Lebenserwartung haben.

\section{Erwartete Innovation}

Bisher war die genetische Diagnostik teuer und benötigte viel Zeit. Zur Identifikation von Mutationen musste die Sanger Methode zum Durchsequenzieren von Zielgenen eingesetzt werden. Daher musste ein hoher Aufwand betrieben werden, um solche Patienten zu finden, die mit hoher Wahrscheinlichkeit tatsächlich an einer sehr seltenen genetisch determinierten Krankheit leiden. Gegenwärtig befindet sich allerdings die genetische Diagnostik im Umbruch. „Gen Panels“ werden zu vergleichsweise niedrigen Preisen angeboten und erlauben das gleichzeitige Screening auf eine Vielzahl von genetischen Krankheiten in kurzer Zeit und mit geringen Kosten. Solche „Gen Panels“ werden in immer mehr Variationen und immer umfangreicher verfügbar. Entsprechend ist eine boomende Industrie in diesem Bereich zu erwarten. Vielfach sind die Sensitivität und Spezifität dieser Gen Panels noch nicht perfekt, aber die Fortschritte in diesem Bereich beschleunigen sich von Jahr zu Jahr. Sie werden zur Identifikation von weit mehr genetisch bedingten Krankheiten führen, als wir uns gegenwärtig vorstellen können.

Die „tailored therapies“ für genetisch verursachte Krankheiten sind teilweise klinisch sehr erfolgreich. So entwickeln bestimmte Genotypen der Cystischen Fibrose bei frühzeitiger Therapie gar keine klinischen Krankheitszeichen mehr. Weitere genetisch gezielt wirksame Medikamente werden mit zunehmender Geschwindigkeit neu entwickelt. Diese Entwicklung ist trotz der großen klinischen Fortschritte, die dadurch für den einzelnen Patienten zu erwarten sind, durch die zu erwartenden enormen Kosten für die Finanzierbarkeit des Gesundheitssystems insgesamt problematisch.

\section{Versorgung aus Patientensicht}

Aus der Sicht eines von einer „orphan disease“ Betroffenen oder seines Angehörigen sind die Wünsche klar: man hofft auf eine wirksame Therapie mit minimalen Nebenwirkungen und eine normale Lebenserwartung. Die Kosten sind erst dann ein unangenehmes Thema, wenn die Krankenversicherung diese nicht er- 
statten möchte oder wenn sich ein großer öffentlicher Druck gegen den Betroffenen richtet. Bei den Nichtbetroffenen, bei Betroffenen mit anderen „orphan diseases“, bei chronisch Kranken mit „non-orphan diseases“ oder manchmal sogar aus den Reihen der Kostenträger hört man immer wieder, dass es im Sinne der Versorgung der gesamten Bevölkerung nicht gerecht sei, wenn die Therapie eines einzelnen Patienten weit mehr koste, als die Therapie von z.B. 100 anderen Patienten gemeinsam. Diese Diskussion wird sich verschärfen, wenn es mehr Indikationen für immer mehr hochpreisige „orphan drugs“ gibt, und sich der finanzielle Spielraum für Krankenversicherer damit verkleinert.

Während in den USA und Großbritannien bereits vor Jahren ein gesellschaftlicher Konsens erzielt wurde, dass es gewisse Obergrenzen bei den Kosten für medizinische Innovationen geben sollte, gibt es in Österreich dazu bislang keinen öffentlichen Diskurs. In vielen Ländern ist es normal, im Zusammenhang mit einer neuen Diagnostik oder Therapie auch die gesundheitsökonomischen Aspekte zu diskutieren. Da$\mathrm{zu}$ ist die Anwendung gesundheitsökonomischer Berechnungsmodelle erforderlich. Eine der etablierten Methoden ist die Berechnung der Quality Adjusted Life Years (QALY), die mit einer bestimmten diagnostischen oder therapeutischen Maßnahme zu gewinnen sind. Damit lassen sich dann ganz einfach die Kosten pro QALY berechnen (= Jahrestherapiekosten/QALY). In keinem Land gibt es strikte Kostengrenzen für die Erstattung einer Maßnahme durch das öffentliche Gesundheitssystem, in den USA werden aber nur selten Maßnahmen erstattet, die mehr als \$100.000 pro QALY kosten, in Großbritannien liegt diese Marke bei etwa GBP 50.000 pro QALY.

\section{Szenario 2025 und Maßnahmenkatalog}

Viel mehr Krankheiten, als uns dies heute bekannt ist, haben einen molekularen Mechanismus. Sehr viel mehr seltene Krankheiten als heute bekannt sind, haben eine genetische Grundlage. Für viele seltene Krankheiten stehen heute „Tailored therapies“ zur Verfügung, die unter hohen Kosten speziell entwickelt wurden.

„Tailored therapies“ können nicht nur das Fortschreiten einer Krankheit aufhalten, sondern auch ihre klinische Manifestation verhindern. Die Betroffenen entwickeln im besten Fall lebenslang keine Krankheitsmerkmale, benötigen aber eine lebenslange Therapie. „Tailored therapies“, viele davon im präventiven Bereich, verbrauchen einen großen Teil der Gesundheitsressourcen. In Zukunft wird es massive Auseinandersetzungen über die Frage geben, welche dieser Therapien erstattet werden sollen und welche nicht.

Nachfolgende Maßnahmen sollen im Zusammenhang von „orphan diseases“ von der ÖGP diskutiert, und falls befürwortet umgesetzt werden:
- öffentlicher Diskurs über die Entwicklung der Kosten der Gesundheit und die ökonomischen Grenzen.

- Lobbyarbeit bei Kostenträgern, um innovative und klinisch hocheffiziente Therapien anbieten zu können, nachdem sie zugelassen wurden.

- Lobbyarbeit bei der Pharmaindustrie, um die Preise der innovativen Medikamente für ,orphan diseases ‘ deutlich zu senken.

- im Bereich der seltenen Erkrankungen durch Pharma-unabhängige Forschung die Abhängigkeit von der Industrie verringern. Dies ist eine vorrangige Aufgabe von öffentlich geförderten Universitäten und Forschungseinrichtungen sowie eine Aufgabe von Forschungsförderungsinstrumenten des Staates

- Shared Risk Modelle (Entwickler und Kostenträger) in Österreich etablieren, um Medikamente nach der Zulassung zu akzeptablen Preisen anbieten zu können.

Orphanet ist eine Datenbank, die Ressourcen zu seltenen Krankheiten und Medikamenten zur Behandlung seltener Krankheiten („orphan drugs“) bündelt, um deren Diagnose und Therapie zu verbessern. Die Entwicklung dieser Datenbank wurde 1997 durch das französische Gesundheitsministerium und das Institut national de la santé et de la recherche médicale (INSERM) initiiert und wird heute von einem Konsortium europäischer Partnerländer unter französischer Federführung mit Förderung durch die Europäische Union betrieben (www.orpha.net/national/ AT-DE/index/über-orphanet/).

Danksagung Wir bedanken uns beim Sekretariat der ÖGP, das diesen Prozess in exzellenter Weise unterstützt hat. Bei Mag. Purzner, der uns über Jahre als Moderator begleitet hat, und ohne dessen Beharrlichkeit dieses Treffen und seine schriftliche Umsetzung wohl nie stattgefunden hätten. Vielen Dank auch allen Mitgliedern der ÖGP und den Vertretern der pneumologischen Selbsthilfegruppen, die durch ihre Teilnahme in Mauerbach das Gelingen ermöglicht haben: Bruckbauer Magdalena, Cima Katharina, Dr., Czerny Marie-Theres, Dr., Dvorak Anton, Dr., Flachberger Hubert, Haider Anna, Dr., Handzhiev Sabin, Dr., Hilbert Markus, Huber Brigitte, Kastner Manuel, Dr., Lechner Maria, Merkle Monika, Dr., Messner Joze, Dr., Nader Astrid, Dr., Renner Andreas, Schandl-Freimüller Petra, Schleicher Verena, Schmid Ingrid, Schneeberger Hermine, Schrittwieser Markus, Stadlbauer Gabriele, Stein Markus, Dr., Stiller Manuela, Dr., Strauss Michaela, Thomüller Ingrid, Dr., Valda Jeanette, Weinhofer Barbara, Waibel Karl, Dr., Zawichowski Doris, Zwick Ralf Harun, Dr. Vertreter der pneumologischen Selbsthilfegruppen: Hofbauer Erika (SH Herz- und Lungentransplantierte), Hoza Josef (SHG Schlafapnoe), Jodlbauer-Riegler Elisabeth (CF Hilfe OÖ), Leeb Elisabeth (IG AV), Nettel Hubert (SH Lungenunion), Oberwaldner Beatrice (med. Beirätin CF Austria), Plattner Ilse (SH Asthma, Allergie und COPD OÖ), Stuppner Bernd (SH Alpha-1-AntitrypsinMangel).

Funding Open access funding provided by Paracelsus Medical University. 
Interessenkonflikt M. Studnicka, B. Baumgartner, J. Bolitschek, D. Doberer, E. Eber, J. Eckmayr, S. Hartl, P. Hesse, P. Jaksch, E. Kink, M. Kneussl, B. Lamprecht, H. Olschewski, A. Pfleger, W. Pohl, C. Prior, C. Puelacher, A. Renner, W. Steflitsch, I. Stelzmüller, H. Täubl, K. Vonbank, M. Wagner, F. Wantke und R. Wass geben an, dass kein Interessenskonflikt besteht.

Open Access Dieser Artikel wird unter der Creative Commons Namensnennung 4.0 International Lizenz veröffentlicht, welche die Nutzung, Vervielfältigung, Bearbeitung, Verbreitung und Wiedergabe in jeglichem Medium und Format erlaubt, sofern Sie den/die ursprünglichen Autor(en) und die Quelle ordnungsgemäß nennen, einen Link zur Creative Commons Lizenz beifügen und angeben, ob Änderungen vorgenommen wurden.

Die in diesem Artikel enthaltenen Bilder und sonstiges Drittmaterial unterliegen ebenfalls der genannten Creative Commons Lizenz, sofern sich aus der Abbildungslegende nichts anderes ergibt. Sofern das betreffende Material nicht unter der genannten Creative Commons Lizenz steht und die betreffende Handlung nicht nach gesetzlichen Vorschriften erlaubt ist, ist für die oben aufgeführten Weiterverwendungen des Materials die Einwilligung des jeweiligen Rechteinhabers einzuholen.

Weitere Details zur Lizenz entnehmen Sie bitte der Lizenzinformation auf http://creativecommons.org/licenses/by/4. 0/deed.de.

\section{Literatur}

\section{Grundlagen für den Masterplan der Pneumologie}

1. Dahlgren G, Whitehead M. 1991. Policies and Strategies to Promote Social Equity in Health. Stockholm: Institute for Futures Studies.

2. Respiratory Society. Forum of respiratory disease. 2. Aufl. Sheffield:Respiratory Society; 2017.

3. GBD 2015 Chronic Respiratory Disease Collaborators. GBD 2015 Chronic Respiratory Disease Collaborators: Global, regional, and national deaths, prevalence, disability-adjusted life years, and years lived with disability for chronic obstructive pulmonary disease and asthma, 1990-2015: a systematic analysis for the Global Burden of Disease Study 2015. LancetRespir Med.2017;5:691-706.

4. Ludwig-Boltzmann-Institut für Suchtforschung. Rauchverhalten in Österreich: Ergebnisse unterschiedlicher aktueller Quellen einschließlich der österreichweiten repräsentativen Bevölkerungsumfrage BMGF/LBISucht/ market. 2008.

5. World Health Organisation. Data and statistics.. http:// www.euro.who.int/en/health-topics/disease-prevention/ tobacco/data-and-statistics. Zugegriffen:28. Okt. 2018.

6. Department of Health. The health consequences of smoking-50 years of progress: a report of the surgeon general. Atlanta: U.S. Department of Health and Human Services, Centers for Disease Control and Prevention, National Center for Chronic Disease Prevention and Health Promotion, Office on Smoking and Health; 2014.

7. McEvvoy CT, Spindel ER. Pulmonary effects of maternal smoking on the fetus and child: effects on lung development, respiratory morbidities, and life long lung health. Paediatr Respir Rev. 2017;21:27-33.
8. Samet JM. Tobacco smoking: the leading cause of preventablediseaseworldwide. ThoracSurg Clin.2013;23(2):103-12. https://doi.org/10.1016/j.thorsurg.2013.01.009.

9. Humair JP, Garin N, Gerstel E, et al. Acute respiratory and cardiovascular admissions after a public smoking ban in Geneva, Switzerland. plos One. 2014;9(3):e90417. https:// doi.org/10.1371/journal.pone.0090417.

10. Hopkinson NS, Hart N, Jenkins G, et al. Climate change and lung health: the challenge for a new president. Throax 2017;72:295-96.

11. D'Amato G, et al. Climate change and respiratory diseases. Eur Respir Rev. 2014;23:161-9.

12. EEA. Air quality in Europe-2018 report. 2018.

\section{Asthma bronchiale und Allergie}

13. GINA-Guidelines: www.gina.arg. 2018. Zugegriffen: 04.09. 2020.

14. S2k-Leitlinie zur Diagnostik und Therapie von Patienten mit Asthma, AWMF-Registernummer 020-009.https:// doi. org/10.1016/j.rmed.2012.12.017.Epub2013 Jan 18.

15. Buhl R, Bals R, Baur X, et al. S2k-Leitlinie zur Diagnostik und Therapie von Patienten mit Asthma. Pneumologie. 2017;71:849-919.

16. GrieblerR, WinklerP, GaiswinklerS, DelcourJ, Juraszovich B, Nowotny M, Pochobradsky E, Schleicher B, Schmutterer I. Österreichischer Gesundheitsbericht. 2016.

17. Statistik Austria, Bundesanstalt für Statistik Österreich. Jahrbuch der Gesundheitsstatistik. 2014.

18. Groos S, Kretschmann J, et al. Nordrheinische Gemeinsame Einrichtung Disease-Management-Programme, Qualitätssicherungsbericht. Disease-Management-Programme in Nordrhein. 2015.

19. Bundesärztekammer (BÄK), Kassenärztliche Bundesvereinigung (KBV), Arbeitsgemeinschaft der Wissenschaftlichen Medizinischen Fachgesellschaften (AWMF). Nationale Versorgung-Leitlinie Asthma - Langfassung. 3. Auflage, 2018. Version 1.AWMF-Register-Nr.: nvl-002. www.asthma. versorgungsleitlinien.de.

20. Aaron SD, Boulet LP, Reddel HK, Gershon AS. Underdiagnosis and overdiagnosis of asthma. Am J Respir Crit Care Med. 2018;198:1012-20.

21. Horak F, Doberer D, Eber E, et al. Diagnosis and management of asthma-statement on the 2015 GINA guidelines. Wien Klin Wochenschr. 2016;128:541-54.

22. LIAISON Study Group, Braido F, Brusselle G, et al. Determinants and impact of suboptimal asthma control in Europe: The INTERNATIONAL CROSS-SECTIONAL AND LONGITUDINAL ASSESSMENT ON ASTHMA CONTROL (LIAISON) study. Respir Res. 2016;17:51.

23. Bruurs ML, van der Giessen LJ, Moed H. The effectiveness of physiotherapy in patients with asthma: a systematic review of the literature. Respir Med. 2013;107:483-94.

24. Zuberbier T, Bachert C, Bousquet PJ, Passalacqua G, Canonica WG, Merk H, et al. GA² LEN/EAACI pocket guide for allergen-specific immunotherapy for allergic rhinitis and asthma. Allergy. 2010;65:1525-30.

25. Virchow JC, Backer V, Kuna P, et al. Efficacy of a house dust mite sublingual allergen immunotherapy tablet in adults with allergic asthma: a randomized clinical trial. JAMA. 2016;315:1715-27.

26. Bousquet J, Arnavielhe S, Bedbrook A, et al. MASK 2017: ARIA digitally-enabled, integrated, person-centred care for rhinitis and asthma multimorbidity using real-worldevidence. Clin Transl Allergy. 2018;8:45. 
27. Siroux V, Ballardini N, Soler M, et al. The asthma-rhinitis multimorbidity is associated with IgE polysensitization in adolescents and adults. Allergy. 2018;73:1447-58.

28. Bousquet J, Heinzerling L, Bachert C, et al. Global Allergy and Asthma European Network: allergic rhinitis and its impact on Asthma. Practical guide to skin prick tests in allergy to aeroallergens. Allergy. 2012;67:18-24.

29. Bousquet J, Arnavielhe S, Bedbrook A, et al. MASK 2017. ARIA digitally-enabled, integrated, person-centred care for rhinitis and asthma multimorbidity using real-worldevidence. Clin Transl Allergy. 2018;8:45.

30. SchultzK, SeidlH,JelusicD, etal.Effectiveness of pulmonary rehabilitation for patients with asthma: study protocol of a randomized controlled trial (EPRA). BMC Pulm Med. 2017;17:49.

31. Karaulov AV, Garib V, Valenta R. Protein biomarkers in Asthma. Int Arch Allergy Immunol. 2018;175:189-208.

32. Anto JM, Bousquet J, Akdis M, et al. Mechanisms of the Development of Allergy (MeDALL): introducing novel concepts in allergy phenotypes. J Allergy Clin Immunol. 2017;139:388-99.

33. Fitzpatrick AM, Moore WC. Severe Asthma phenotypes-how should they guide evaluation and treatment? JAllergy Clin Immunol Pract. 2017;5:901-8.

\section{COPD}

34. Hsien-Ho L, Cohen MT, et al. Effects of smoking and solidfuel use on COPD, lung cancer, and tuberculosis in China: a time-based, multiple risk factor, modelling study. Lancet. 2008;372(9648):1473-83.

35. Firlei N, Lamprecht B, Schirnhofer L, etal. Die Prävalenz der COPD in Österreich - die erwartete Entwicklung bis 2020. Wien Klin Wochenschr. 2007;119(17-18):513-8.

36. Ludwig Boltzmann Institute for COPD and Respiratory Epidemiology. The Austrian LEAD study. Ludwig Boltzmann institute for COPD and respiratory epidemiology. 2019. www.leadstudy.at.

37. NowakP, GeißlerW, Holzer U, etal. Themenqualitätsbericht COPD 2013. Wien: Bundesinstitut für Qualität im Gesundheitswesen, Bundesministerium für Gesundheit; 2013.

38. Pock M, Czypionka T, Reiss M, Röhrling G. Economic Burden of Chronic Respiratory Diseases in Austria and Slovenia: Results of a life-cycle model. Projektbericht. Wien: Institut für Höhere Studien (IHS). 2018, https://www.ihs.ac.at/de/ueber-uns/pr/news/; http:// irihs.ihs.ac.at/4663/1/2018-ihs-report-pock-czypionkareiss-copd-chronic-respiratorydiseases-austria-slovenia. pdf.Zugegriffen:04.09.2020.

39. Hartl S, Lopez-Campos JL, Pozo-Rodriguez F, et al. Risk of death and readmission of hospital-admitted COPD exacerbations: European COPD Audit. Eur Respir J. 2016;47(1):113-21. https://doi.org/10.1183/13993003. 01391-2014.

40. Postma DS, Bush A, van den Berge M. Risk factors and early origins of chronic obstructive pulmonary disease. Lancet. 2015;385(9971):899-909. https://doi.org/10.1016/ S0140-6736(14)60446-3.

41. Ring B, BurbankAJ, Mills K, Ivins S, Dieffenderfer J, Hernandez ML. Validation of an app-based portable spirometer in adolescents with asthma. J Asthma. 2019; https://doi.org/ 10.1080/02770903.2019.1702201.

\section{Mukoviszidose (Cystische Fibrose, CF) und andere Krankheitsbilder der pädiatrischen Pneumologie}

42. Orenti A, Zolin A, Nährlich L, van Rens J, et al. ECFSPR Annual Report2016.2018.

43. Burgel PR, Bellis G, Olesen HV, Viviani L, Zolin A, Blasi F, et al. Future trends in cystic fibrosis demography in 34 European countries. Eur Respir J.2015;46:133-41.

44. Jaksch P, Ankersmit J, Scheed A, et al. Alemtuzumab in lung transplantation: an open-label, randomized, prospective single center study. Am J Transplant. 2014;14(8):1839-45.

45. Hoetzenecker K, Schwarz S, Muckenhuber M, et al. Intraoperative extracorporeal membrane oxygenation and the possibility of postoperative prolongation improve survival in bilateral lung transplantation. J Thorac Cardiovasc Surg. 2018;155(5):2193-2206.e3.

46. Weiss S, Van Egmont-Froehlich A, Hofer N, et al. Long-term respiratory support for children and adolescents in Austria: a national survey. Klin Padiatr. 2016;228:42-6.

47. Lucas JS, Barbato A, Collins SA, et al. European Respiratory Society guidelines for the diagnosis of primary ciliary dyskinesia. Eur Respir J. 2017;49(1):1601090. https://doi.org/10. 1183/13993003.01090-2016.

48. Baraldi E, Filippone M. Chronic lung disease after premature birth. NEngl J Med. 2007;357:1946-55.

49. Davenport M, Eber E. Long term respiratory outcomes of congenital thoracic malformations. Semin Fetal Neonatal Med.2012;17:99-104.

\section{Infektionen in der Pneumologie}

50. ERS. The European lung white book, respiratory health and disease in Europe. 2013.

51. Peters E, Pritzkuleit R, Beske F, Katalinic A. Demographic changeand diseaserates: aprojection until2050. BundesgesundheitsblattGesundheitsforschung Gesundheitsschutz. 2010;53(5):417-263.

52. Chalmers JD, Pletz MW, Aliberti S. Communitiy-Acquired Pneumoniain European Respiratory Monograph 63. March 2014.

53. S3-Leitlinie Behandlung von erwachsenen Patienten mit ambulant erworbener Pneumonie und Prävention - Update 2016

54. Floto RA, Haworth CS. Bronchiectasis in European Respiratory Monograph 52, June. 2011.

55. Polverino E, Goeminne PC, McDonnell MJ, et al. European Respiratory Society guidelines for the management of adult bronchiectasis. Eur Respir J. 2017;50(3):1700629. https:// doi.org/10.1183/13993003.00629-2017.

56. EMBARC-The European Bronchiektasis Registry (zitiert am 10. Dez. 2017).

57. PROGNOSIS-The Prospective German Non-CF-Bronchiectasis Patient Registry (zitiert am 10. Dez. 2017).

58. WHO. Global Tuberculosis Report. 2017.

59. AGES. Tuberkulose-Jahresbericht. 2016.

60. Schaberg T, Bauer T, Sk-Leitlinie BF. Tuberkulose im Erwachsenenalter. Stuttgart: Thieme; 2017.

61. RIS-Tuberkulosegesetz-Bundesrechtkonsolidiert (eingesehen am 10. Dez. 2017).

62. Diel R, Vandeputte J, de Vries G, Stillo J, Wanlin M, Nienhaus A. Costs of tuberculosis disease in the European Union: a systematic analysis and cost calculation. Eur Respir J. 2014;43(2):554-65. https://doi.org/10.1183/ 09031936.00079413. 


\section{Schlafbezogene Atmungsstörungen}

63. Garvey JF. Epidemiological aspects of obstructive sleep apnea. J Thorac Dis. 2015;7(5):920-92.

64. Heinzer R, Vat S, Marques-Vidal P, Marti-Soler H, et al. Prevalence of sleep-disordered breathing in the general population: the HypnoLaus study. Lancet Respir Med. 2015;3(4):310-8.

65. Mayer G, Arzt M, Braumann B, Ficker JH, et al. Nürnberg S3-Leitlinie Nicht erholsamer Schlaf/Schlafstörungen - Kapitel „Schlafbezogene Atmungsstörungen“. Springer Medizin: Deutsche Gesellschaft für Schlafforschung und Schlafmedizin (DGSM); 2017.

66. Apneuvereiniging. Apneu Magazin. 2014.

67. Huppmann M. Empfehlungen zur ambulanten Polygraphie des AK der ÖGP. 2007. https://www.ogp.at/media/ publikationen/Empfehlung_ambulante_Polygraphie.pdf. Wien Klin Wochenschr. 2007;119/9-10:326-30. https://doi. org/10.1007/s00508-007-0800-9.

68. Landesrechnungshof Vorarlberg. Prüfungsbericht Vorarlberger Spitalswesen, November 2009 bis März 2010.2010.

69. Rejón-Parrilla JC, Garau M, Sussex J. Obstructive Sleep Apnoea Health Economics Report, Office of Health Economics, September 2014. London, UK. NHS North of England Specialised Commissioning Group. 2012. https:/ / www.blf. org.uk/sites/default/files/OHE-OSA-health-economicsreport---FINAL---v2.pdf.

70. Multidiscipliair benadering. Multidisciplinair teambesprekingen Rapport slapklinieken 2016:26-27. 2016. http:// apneuvereniging.todw.nl/Apneu_TNS_rapport_2016.pdf. Zugegriffen:04.09.2020.

71. Apneuveriniging. Diagnose en behandeling van apneu in slaapklinieken. Apneu magazin. 2014:15. 2014. http://apneuvereniging.todw.nl/20141114_Apneu_TNS_ rapport.pdf.Zugegriffen: 04.09.2020.

72. Case Management in Österreich und Europa. http://www. hauptverband.at/cdscontent/load?contentid $=10008.59798$ 1\&version=1397024310. Zugegriffen: 04.09.2020.

73. Tragbare Datenverarbeitung. https://de.wikipedia.org/w/ index.pho?title=Wearable_Computing\&oldid=202528655. Zugegriffen:04.09.2020.

74. Patzak A. Continuous blood pressure measurement using the pulse transit time: comparison to intra-arterial measurement. Blood Press. 2015;24(4):217-21.

75. Riedl AM. ÖÄK Lehrgang Geriatrie - Abschlussarbeit Obstruktives Schlafapnoe-Syndrom. : Akademie der Ärzte.

76. PolsekN.ObstructivesleepapnoeaandAlzheimer's disease: in search of shared pathomechanisms. Neurosci Biobehav Rev. 2018;86:142-9.

77. Bliwise DL. Epidemiology of age-dependence in sleep disordered breathing (SBD) in old age: the Bay Area Sleep Cohort (BASC). Sleep Med Clin. 2009;4(1):57-64.

78. Haruki N, Floras JS. Sleep-Disordered Breathing in Heart Failure-A Therapeutic Dilemma. Circ J. 2017;81(7):903-12. https://doi.org/10.1253/circj.CJ-17-0440.

79. Onen F, Onen H. Obstructive sleep apnea and cognitive impairment in the elderly. Psychol Neuropsychiatr Vieil. 2010;8(3):163-9.

80. Framnes SN, Arble DM. The bidirectional relationship between obstructive sleep apnea and metabolic disease. Front Endocrinol. 2018;9:440.

81. Owens RL, Gold KA, Gozal D, et al. Sleep and breathing ... and cancer? Prev Res. 2016;9(11):821-7.

82. Farre N, Farre R, Gozal D. Sleep apnea morbidity A consequence of microbial-immune crosstalk. Chest. 2018;154(4):754-9. https://doi.org/10.1016/j.chest.2018. 03.001 .
83. Cappucio FP, D’Elia L, Strazzullo P, Miller MA. Quantity and quality of sleep and incidence of type 2 diabetes: a systematic review and meta-analysis. Diabetes Care. 2010;33(2):414-20.https://doi.org/10.2337/dc09-1124.

84. Valipour A, Lothaller H, Rauscher H, et al. Gender-related differences in the obstructive sleep apnea syndrome. Pneumologie. 2012;66(10):584-8.

85. Simpson R, Oyekan AA, Ehsan Z, Ingram DG. Obstructive sleep apnea in patients with Down syndrome: current perspectives. NatSciSleep. 2018;10:287-93.

86. Randerath WD, Sandhya G. Opioid-induced sleep apnea: is it a real problem? J Clin Sleep Med. 2012;8(5):577-8.

87. Leitlinie für die gesundheitliche Eignung von Kraftfahrzeuglenkern https://www.bmvit.gv.at/verkehr/strasse/ recht/fsg/erlaesse/downloads/gesundheit_leitlinien.pdf. Zugegriffen: 04.09.2020.

88. Irer B, Çelikhisar A, Çelikhisar H, Bozkurt O, Demir Ö. Evaluation of sexual dysfunction, lower urinary tract symptoms and quality of life in men with obstructive sleep apnea syndrome and the efficacy of continuous positive airway pressure therapy. Urology. 2018;121:86-92.

89. Jurado-Gomez B, Guglielmi O, Gude-Sampedro F. Effect of CPAP therapy on job productivity and psychosocial occupational health in patients with moderate to severe sleep apnea. Sleep Breath. 2015;19(4):1293-9.

90. Nederlandse Zorgautoriteit. DBC zorgproducten \& zorgactiviteiten. 2015.

\section{Interventionelle Pneumologie}

91. Statistik Austria. Österreichisches Krebsregister (Stand 19.12.2018) und Todesursachenstatistik. 2018.

92. Oki M, Saka H, Kitagawa C, Kogure Y, Murata N, Adachi T, et al. Rapid on-site cytologic evaluation during endobronchial ultrasound-guided transbronchial needle aspiration for diagnosing lung cancer: a randomized study. Respiration. 2013;85(6):486-92.https://doi.org/10.1159/000346987.

93. Nakajima T, Yasufuku K, Saegusa F, Fujiwara T, Sakairi Y, HiroshimaK, etal.Rapidon-sitecytologic evaluation during endobronchial ultrasound-guided transbronchial needle aspiration for nodal staging in patients with lung cancer. Ann Thorac Surg. 2013;95:1695-9.

94. Gu P, Zhao YZ, Jiang LY, Zhang W, Xin Y, Han BH. Endobronchial ultrasound-guided transbronchial needle aspiration for staging of lung cancer: a systematic review and metaanalysis. Eur J Cancer. 2009;45:1389-96.

95. van Agteren J, Hnin K, Grosser D, Carson K, BJ S. Bronchoscopic lung volume reduction procedures for chronic obstructive pulmonary disease. Cochrane Database Syst Rev. 2017; https://doi.org/10.1002/14651858.CD012158.pub2.

96. Kemp S, Slebos D, Kirk A, Kornaszewska M, Carron K, Ek L, et al. A multicenter randomized controlled trial of zephyr endobronchial valve treatment in heterogeneous emphysema (TRANSFORM). Am J Respir Crit Care Med. 2017;196(12):1535-1534.

97. Gompelmann D, Eberhard R, Herth F. Endoskopische Volumenreduktion bei COPD - eine kritische Bestandsaufnahme. Dtsch Arztebl. 2014;111(49):827-33.

98. Fiorelli A, Santoriello C, De Felice A, Ferrigno F, Carlucci A, De Ruberto E, et al. Bronchoscopic lung volume reduction with endobronchial valves for heterogeneous emphysema: long-term results. J Visc Surg. 2017;3:170. https://doi.org/ 10.21037/jovs.2017.10.04. 


\section{Thorakale Onkologie}

99. Pritzkuleit R, Beske F, Katalinic A. Erkrankungszahlen in der Pneumologie - eine Projektion bis 2060. Pneumologie. 2010;64:535-40.

100. The National Lung Screening Trial Research Team. Reduced lung-cancer mortality with low-dose computed tomographic screening. N Engl J Med.2011;365(5):395-409. https:/ / doi.org/10.1056/NEJMoal102873.

101. Pastorino U, Boffi R, Marchianò A, Sestini S, et al. Stopping smoking reduces mortality in low-dose computed tomography screening participants. J Thorac Oncol. 2016;11(5):693-9.

102. Tanner NT, Kanodra NM, Gebregziabher M, et al. The association between smoking abstinence and mortality in the national lung screening trial. Am J Respir Crit Care Med. 2016;193(5):534-41.

103. de Koning HJ, van der Aalst CM, de Jong PA, et al. Reduced lung-cancer mortality with volume CT screening in a randomized trial. n Engl J Med. 2020;382(6):503-13. https:// doi.org/10.1056/NEJMoa1911793.

104. Prävention, Diagnostik, Therapie und Nachsorge des Lungenkarzinoms. Interdisziplinäre S3-Leitlinie der Deutschen Gesellschaft für Pneumologie und Beatmungsmedizin und der Deutschen Krebsgesellschaft. Stand: 28.02.2018, gültig bis31.Dez. 2022

105. IHE Comparator Report 2016, IQVIA 2017, Pharmig FactsheetOnkologie

\section{Orphan Diseases (seltene Krankheiten)}

106. Hinks TS, ZhouX, Staples KJ, etal. Innateand adaptiveT cells in asthmatic patients: relationship to severity and disease mechanisms. J Allergy Clin Immunol. 2015;136:323-33.

107. Kovacs G, Agusti A, Barbera JA, et al. Pulmonary vascular involvement in COPD-is there a pulmonary vascular phenotype? Am J Respir Crit Care Med. 2018; https://doi.org/ 10.1164/rccm.201801-0095PP.

108. de Koning HJ, van der Aalst CM, de Jong PA, et al. Reduced lung-cancer mortality with volume CT screening in a randomized trial. N Engl J Med. 2020;382(6):503-13. https:// doi.org/10.1056/NEJMoa1911793.
109. Hoyles RK, Ellis RW, Wellsbury J, et al. A multicenter, prospective, randomized, double-blind, placebo-controlled trial of corticosteroids and intravenous cyclophosphamide followed by oral azathioprine for the treatment of pulmonary fibrosis in scleroderma. Arthritis Rheum. 2006;54:3962-70.

110. McCormack FX, Gupta N, Finlay GR, et al. Official American Thoracic Society/Japanese Respiratory Society clinical practice guidelines: lymphangioleiomyomatosis diagnosis and management. Am J Respir Crit Care Med. 2016;194:748-61.

111. Borie R, Danel C, Debray MP, et al. Pulmonary alveolar proteinosis. Eur Respir Rev. 2011;20:98-107.

112. Galie N, Humbert M, Vachiery JL, et al. 2015 ESC/ERS Guidelines for the diagnosis and treatment of pulmonary hypertension: The Joint Task Force for the Diagnosis and Treatment of Pulmonary Hypertension of the European Society of Cardiology (ESC) and the European Respiratory Society (ERS): Endorsed by: Association for European Paediatric and Congenital Cardiology (AEPC), International Society for Heart and Lung Transplantation (ISHLT). Eur Respir J.2015;46:903-75.

113. Martinez FJ, Collard HR, Pardo A, et al. Idiopathic pulmonary fibrosis. Nat Rev Dis Primers. 2017;3:17074.

114. Kwapiszewska G, Gungl A, Wilhelm J, et al. Transcriptome profiling reveals the complexity of pirfenidone effectsin IPF. Eur Respir J. 2018;52(5):1800564. https://doi.org/10.1183/ 13993003.00564-2018.

115. Reinmuth N, Gröschel A, Schumann C, Sebastian M, Wiewrodt R, Reck M. Therapy of metastatic non-small cell lung cancer. Pneumologie. 2016;70:567-78.

116. Rolfo C, Mack PC, Scagliotti GV, et al. Liquid biopsy for advanced non-small cell lung cancer (NSCLC): a statement paper from the IASLC. J Thorac Oncol. 2018;13:1248-68.

117. Fitzgerald DA, Doumit M, Abel F. Changing respiratory expectations with the new disease trajectory of nusinersen treated spinal muscular atrophy [SMA] type 1. Paediatr Respir Rev. 2018;28:11-7.

118. Scoto M, Finkel R, Mercuri E, Muntoni F. Genetic therapies for inherited neuromuscular disorders. Lancet Child Adolesc Health. 2018;2:600-9.

Hinweis des Verlags Der Verlag bleibt in Hinblick auf geografische Zuordnungen und Gebietsbezeichnungen in veröffentlichten Karten und Institutsadressen neutral. 